JOURNAL OF THE

AMERICAN MATHEMATICAL SOCIETY

Volume 12, Number 1, January 1999, Pages 145-171

S 0894-0347(99)00283-0

\title{
GLOBAL WELLPOSEDNESS OF DEFOCUSING CRITICAL NONLINEAR SCHRÖDINGER EQUATION IN THE RADIAL CASE
}

\author{
J. BOURGAIN
}

\section{INTRODUCTION}

In this paper, we show that the initial value problem (IVP) for the nonlinear Schrödinger equation (NLS) in 3 space dimensions (3D)

$$
\left\{\begin{array}{l}
i u_{t}+\Delta u-u|u|^{4}=0 \\
u(0)=\phi \in H^{1}\left(\mathbb{R}^{3}\right), \phi \text { radial }
\end{array}\right.
$$

is globally wellposed in time. More precisely, we obtain a unique solution $u=u_{\phi} \in$ $\mathcal{C}_{H^{1}}([0, \infty[)$ such that for all time, $u(t)$ depends continuously on the data $\phi$ (in fact, the dependence is even real analytic here). Moreover, there is scattering for $t \rightarrow \infty$. The same statement holds for radial data $\phi \in H^{s}, s \geq 1$ and proves in particular global existence of classical solutions in the radially symmetric case. Also this issue was open. Thus this is the analogue for NLS of the result for the wave equation with quintic nonlinearity obtained by M. Struwe [Str] in the radial case (and by M. Grillakis [Gr], [S-S], in general). In the case of the wave equation, the proof is based on the following two different facts:

(i) As a consequence of the analysis of the local IVP, if global wellposedness fails, there is necessarily a "concentration" effect of the solution on small balls (that may be centered at 0 in the radial case).

(ii) The Morawetz inequality, which forbids an infinite repetition of the effect described in (i).

The main problem to follow that scheme for (0.1) is due to the fact that the analogue of the Morawetz inequality for NLS (see [L-S]), implying an apriori bound on

$$
\iint \frac{|u|^{6}}{|x|} d x d t
$$

is not sufficient to disprove the concentration effect.

(This is not surprising since (0.2) is in fact already bounded by $\sup _{t}\|u(t)\|_{H^{1 / 2}}^{2}$.)

We will use, however, together with some other ideas, the following variant of Morawetz' inequality obtained basically by localizing the argument.

Received by the editors April 20, 1998.

1991 Mathematics Subject Classification. Primary 35Q55, 35L15.

Key words and phrases. Nonlinear Schrödinger equation, global wellposedness.

(C)1999 American Mathematical Society 
Assume $I$ is some time interval. Then

$$
\int_{I} \int_{|x|<|I|^{1 / 2}} \frac{|u(x, t)|^{6}}{|x|} d x d t<C H(\phi)|I|^{1 / 2} .
$$

Recall that the concentration effect in space-time relates to the $L_{x, t}^{10}$-norm and we get space-time boxes $Q$ of size $\delta \times \delta \times \delta \times \delta^{2}$ where $\delta \rightarrow 0$ such that

$$
\inf \|u\|_{L_{x, t}^{10}(Q)}>0
$$

assuming no global wellposedness. Considering only the spatial variable $x$, there is concentration of $H^{1}$-norm and $L^{6}$-norm on size $\delta$ balls for certain times $t$ (in the setting (0.4), we get a time interval of size $\left.\delta^{2}\right)$.

In order to establish global wellposedness for $(0.1)$, it will suffice to get a uniform bound

$$
\|u\|_{L_{x, t}^{10}[J]}<C
$$

assuming (0.1) wellposed on the time interval $J$.

We prove this by an inductive argument on the size of the Hamiltonian

$$
H(\phi)=\frac{1}{2} \int|\nabla \phi|^{2}+\frac{1}{6} \int|\phi|^{6}
$$

(the property holds provided $H(\phi)$, hence $\|\phi\|_{H^{1}}$, is sufficiently small).

The main points of the argument may be summarized as follows:

(i) Assume

$$
\|u\|_{L_{x, t}^{10}[J]}>M
$$

for some time interval $J$ on which (0.1) is wellposed. Using (0.3) and an argument based on the fact $\|u(t)\|_{H^{1}}$ remains uniformly bounded, one shows the existence of a subinterval $I=\left[t_{0}, b\right] \subset J$ such that

$$
\begin{aligned}
\|u\|_{L_{x, t}^{10}[t \in I]} & =\eta, \\
\left\|\nabla u\left(t_{0}\right)\right\|_{L^{2}\left[|x|<\kappa|I|^{1 / 2}\right]} & >\eta^{3 / 2} .
\end{aligned}
$$

Here $\eta$ is a fixed small number (except for the fact that we let $\eta \rightarrow 0$ if $\|\phi\|_{H^{1}} \rightarrow$ $\infty)$. The number $\kappa$ will be chosen sufficiently small (depending on the induction hypothesis for initial data $\psi$ satisfying say $\left.H(\psi)<H(\phi)-\eta^{4}\right)$ and this is possible provided $M$ in (0.6) is taken large enough.

(ii) Write on $J \cap\left[t_{0}, \infty[\right.$

$$
u=v+w
$$

where $v$ satisfies the IVP

$$
\left\{\begin{array}{l}
i v_{t}+\Delta v-v|v|^{4}=0, \\
v\left(t_{0}\right)=\zeta u\left(t_{0}\right),
\end{array}\right.
$$

and $0<\zeta<1$ is a radial bump function chosen such that

$$
\begin{gathered}
\left\{\begin{array}{l}
\zeta=1 \text { if }|x|<\kappa|I|^{1 / 2}, \\
\zeta=0 \text { if }|x|>C \kappa|I|^{1 / 2},
\end{array}\right. \\
\left\|w\left(t_{0}\right)\right\|_{H^{1}}^{2}=\left\|(1-\zeta) u\left(t_{0}\right)\right\|_{H^{1}}^{2}<\left\|u\left(t_{0}\right)\right\|_{H^{1}}^{2}-\eta^{3},
\end{gathered}
$$

which is possible by $(0.8)$. 
Also, because of (0.7),

$$
\begin{aligned}
& \left\|e^{i\left(t-t_{0}\right) \Delta} u\left(t_{0}\right)\right\|_{L_{x, t}^{10}[t \in I]} \leq \eta, \\
& \left\|e^{i\left(t-t_{0}\right) \Delta} v\left(t_{0}\right)\right\|_{L_{x, t}^{10}[t \in I]} \lesssim \eta, \\
& \|v\|_{L_{x, t}^{10}[t \in I]} \lesssim \eta,
\end{aligned}
$$

and $u, v, w$ behave on $I=\left[t_{0}, b\right]$ essentially according to the linear flow, up to an error at most $\eta^{4}$, i.e.

$$
\left\|w(t)-e^{i\left(t-t_{0}\right) \Delta} w\left(t_{0}\right)\right\|_{H^{1}} \lesssim \eta^{4} \text { for } t \in I
$$

(from $(0.14)$, it follows in particular that $(0.10)$ is wellposed on $I$ ).

From (0.12), (0.16) we deduce that for $t \in I$

$$
\|w(t)\|_{H^{1}}^{2}<\left\|u\left(t_{0}\right)\right\|_{H^{1}}^{2}-\frac{1}{2} \eta^{3}
$$

and also

$$
H(w(t))<H\left(u\left(t_{0}\right)\right)-\frac{1}{2} \eta^{3}=H(\phi)-\frac{1}{2} \eta^{3} .
$$

(iii) Considering $v$ on $\left[t_{0}, b\right]$, the pseudo-conformal conservation law (see [G-V2]) implies in particular an estimate

$$
\left(t-t_{0}\right)^{2}\|v(t)\|_{L_{x}^{6}}^{6} \leq C\left\||x| v\left(t_{0}\right)\right\|_{L_{x}^{2}}^{2}<C \kappa^{2}|I|^{2}
$$

taking (0.11) into account.

Hence, for $t>b$

$$
\|v(t)\|_{L_{x}^{6}}<C \kappa^{1 / 3}<\kappa^{1 / 4} .
$$

It follows in particular that $(0.10)$ is globally wellposed on $\left[t_{0}, \infty\left[\right.\right.$ (if not, $L_{x}^{6}$ concentration effects would need to occur for some times $t>b$, which is impossible since, by (0.20), $\|v(t)\|_{6}$ is in fact small for $\left.t>b\right)$.

(iv) It remains to analyze the behavior of $w$ on $J \cap[b, \infty[$, satisfying the IVP

$$
\left\{\begin{array}{l}
i w_{t}+\Delta w-|v+w|^{4}(v+w)+|v|^{4} v=0 \\
w(b)=(1-\zeta) u(b)
\end{array}\right.
$$

and we compare $w$ with $W$ solving

$$
\left\{\begin{array}{l}
i W_{t}+\Delta W-|W|^{4} W=0, \\
W(b)=w(b) .
\end{array}\right.
$$

Since (0.12) yields a reduction of the Hamiltonian, the inductive hypothesis on the data implies that $(0.22)$ is globally wellposed and an estimate

$$
\|W\|_{L_{x, t}^{10}}<M_{1}
$$

holds for some constant $M_{1}$. The remainder of the argument consists in bounding $W-w$; taking in $(0.20) \kappa$ sufficiently small (depending on $M_{1}$ ), equation (0.21) may indeed be seen as a perturbation of $(0.22)$. The conclusion is that in particular $\|\Gamma\|_{L^{10}[J \cap[b, \infty[]}=o(1)<1, \Gamma=w-W$, which, together with (0.23), is used to contradict $(0.6)$. 
Looking back at inequalities (0.12), (0.16), the (high) order $\left(|u|^{5}\right)$ of the nonlinearity in (0.1) clearly plays a role in the preceding argument. In the case $D=4$, the corresponding equation becomes

$$
i u_{t}+\Delta u-u|u|^{2}=0
$$

with only cubic nonlinearity. In section 7 , we indicate a variant of the method that permits us to treat $(0.24)$ as well. The case of general dimension will be pursued elsewhere.

1.

Consider the IVP for 3D defocusing NLS (in the radial case)

$$
\left\{\begin{array}{l}
i u_{t}-\Delta u+u|u|^{4}=0, \\
u(0)=\phi \in H^{1}\left(\mathbb{R}^{3}\right),
\end{array}\right.
$$

with Hamiltonian

$$
H(u)=\frac{1}{2} \int_{\mathbb{R}^{3}}|\nabla u|^{2}+\frac{1}{6} \int_{\mathbb{R}^{3}}|u|^{6}=H(\phi) .
$$

Our purpose is to prove global wellposedness of (1.1) using an inductive argument on the size of $H(\phi)$; for $H(\phi)$ sufficiently small, this is indeed the case.

\section{Morawetz APRiori inequality (a REFinement)}

Lemma 2.1. If $u$ is a smooth solution of (1.1) on a time interval $I \subset \mathbb{R}$, then

$$
\int_{I} \int_{|x|<|I|^{1 / 2}} \frac{|u(x, t)|^{6}}{|x|} d x d t \leq C H(\phi)|I|^{1 / 2} .
$$

Proof (cf. [L-S], Lemma 4). Write $r=|x|, u=v+i w$. Consider a bump function $\varphi$ on $\mathbb{R}^{3}$ satisfying

$$
\left\{\begin{array}{l}
\varphi=1 \text { for }|x|<\delta \\
\varphi=0 \text { for }|x|>2 \delta \\
\varphi \text { radial, }\left|\varphi^{(j)}\right| \lesssim \delta^{-j}
\end{array}\right.
$$

One has

$$
0=\operatorname{Re}\left(i u_{t}-\Delta u+|u|^{4} u\right)\left(\bar{u}_{r}+\frac{\bar{u}}{r}\right)=\frac{\partial X}{\partial t}+\nabla \cdot Y+Z
$$

where

$$
X=-w\left(v_{r}+\frac{v}{r}\right)
$$

(2.6) $Y=\frac{x}{r} v_{t} w-\nabla v\left(v_{r}+\frac{v}{r}\right)-\nabla w\left(w_{r}+\frac{w}{r}\right)+\frac{x}{2 r}|\nabla u|^{2}+\frac{x}{6 r}|u|^{6}-\frac{x}{2 r^{3}}|u|^{2}$,

(2.7) $Z=\frac{1}{r}\left(|\nabla u|^{2}-\left|u_{r}\right|^{2}\right)+\frac{2}{3 r}|u|^{6}$. 
Multiplying both sides of (2.4) by $\varphi$ and integrating in $(x, t) \in \mathbb{R}^{3} \times I, I=[a, b]$, we get

$$
\begin{aligned}
\int_{\mathbb{R}^{3}}[X(x, b) & -X(x, a)] \varphi(x) d x+\int_{I} \int_{\mathbb{R}^{3}}(\nabla \cdot Y) \varphi d x d t \\
& +\int_{I} \int_{\mathbb{R}^{3}}\left\{\frac{1}{r}\left(|\nabla u|^{2}-\left|u_{r}\right|^{2}\right)+\frac{2}{3 r}|u|^{6}\right\} \varphi d x d t=0 .
\end{aligned}
$$

Since $(\nabla \cdot Y) \varphi=\nabla \cdot(\varphi Y)-\nabla \varphi \cdot Y$, excision of the singularity in the last term of $Y$ in (2.6) gives

$$
\int \nabla \cdot(\varphi Y) d x \sim \varphi(0)|u(0, t)|^{2}=|u(0, t)|^{2} .
$$

Thus (2.8), (2.9) imply in particular that

$$
\frac{2}{3} \int_{I} \int \frac{|u|^{6}}{r} \varphi(r) d x d t \leq \sup _{t \in I}\left|\int X(x, t) \varphi d x\right|+|I| \sup _{t \in I}\left|\int \nabla \varphi Y d x\right| .
$$

By (2.5), (2.3), one has for fixed $t$

$$
\begin{aligned}
\int|X| \varphi d x & =\int\left(|\nabla u|+\frac{|u|}{r}\right)|u| \varphi d x \\
& \leq\left(\|u(t)\|_{H^{1}}+\left\|\frac{u(t)}{r}\right\|_{2}\right)\|u(t) \varphi\|_{2} \\
& \leq C\|u(t)\|_{H^{1}}\|u(t)\|_{6}\|\varphi\|_{3} \\
& \lesssim \delta\|u(t)\|_{H^{1}}^{2} .
\end{aligned}
$$

By (2.3), (2.6)

$$
\begin{aligned}
\left|\int \nabla \varphi \cdot Y d x\right| & \leq\left|\int \varphi^{\prime}(r) v_{t} w\right|+\frac{C}{\delta} \int\left[\left(|\nabla u|+\frac{|u|}{r}\right)^{2}+|u|^{6}\right] \\
& <\left|\int \varphi^{\prime}(r) \Delta w w\right|+\frac{C}{\delta} H(\phi)
\end{aligned}
$$

where

$$
\begin{aligned}
& \left|\int \varphi^{\prime}(r) \Delta w w\right|<\frac{1}{\delta} \int|\nabla u|^{2}+\int\left|\varphi^{\prime \prime}(r)\right||\nabla u||u| \\
& \quad \lesssim \frac{1}{\delta}\|u(t)\|_{H^{1}}^{2}+\frac{1}{\delta^{2}}\|u(t)\|_{H^{1}}\left(\int_{|x|<\delta}|u|^{2} d x\right)^{1 / 2} \lesssim \frac{1}{\delta}\|u(t)\|_{H^{1}}^{2} .
\end{aligned}
$$

Hence

$$
\left|\int \nabla \varphi \cdot Y d x\right| \lesssim \frac{1}{\delta} H(\phi)
$$

Thus from (2.10), (2.11), (2.14)

$$
\int_{I} \int_{|x|<\delta} \frac{|u|^{6}}{r} d x d t \lesssim H(\phi)\left(\delta+\frac{|I|}{\delta}\right)
$$

and letting $\delta=|I|^{1 / 2}$, (2.2) follows. 


\section{A CONCENTRATION PROPERTY}

Our purpose here is to elaborate more on some aspects of the local wellposedness theory for (1.1). Standard references for this issue are the papers $[\mathrm{C}-\mathrm{W}]$ and $[\mathrm{G}-\mathrm{V} 1]$.

Assume (1.1) is wellposed on the time-interval $I=[a, b]$ and

$$
\|u\|_{L_{x, t}^{10}[I]}=\eta
$$

where $\eta$ is a sufficiently small but fixed number.

From the integral equation

$$
u(t)=e^{i(t-a) \Delta} u(a)+i \int_{a}^{t} e^{i(t-\tau) \Delta}\left(u|u|^{4}\right)(\tau) d \tau
$$

it follows that

$$
\begin{aligned}
& \left\|D_{x}^{3 / 5} u\right\|_{L_{t \in I}^{10} L_{x}^{10 / 3}} \leq \\
& \quad\left\|e^{i(t-a) \Delta}\left[D_{x}^{3 / 5} u(a)\right]\right\|_{L_{t}^{10} L_{x}^{10 / 3}} \\
& \quad+\left\|\int_{a}^{t} e^{i(t-\tau) \Delta} D_{x}^{3 / 5}\left[\left.u|u|^{4}\right|_{\tau \in I}\right] d \tau\right\|_{L_{t \in I}^{10} L_{x}^{10 / 3}} .
\end{aligned}
$$

From Strichartz' inequality, estimate

$$
(3.3) \leq C\left\|e^{i(t-a) \Delta}\left[D_{x} u(a)\right]\right\|_{L_{x, t}^{10 / 3}} \leq C\left\|D_{x} u(a)\right\|_{2}=C\|u\|_{H^{1}} .
$$

From the decay of the linear group $e^{i t \Delta}$ in $3 D$

$$
\left\|e^{i t \Delta} \psi\right\|_{\infty} \lesssim|t|^{-3 / 2}\|\psi\|_{1}
$$

and interpolation, it follows that

$$
\left\|e^{i t \Delta} \psi\right\|_{\frac{10}{3}} \lesssim|t|^{-3 / 5}\|\psi\|_{\frac{10}{7}} .
$$

By (3.7)

$$
(3.4) \leq C\left\|\int \frac{1}{|t-\tau|^{3 / 5}}\right\| D_{x}^{3 / 5}\left[\left.u|u|^{4}\right|_{\tau \in I}\right]\left\|_{L_{x}^{10 / 7}} d \tau\right\|_{L_{t}^{10}}
$$

where by estimates on fractional derivatives and Hölder's inequality

$$
\left\|D_{x}^{3 / 5}\left[u|u|^{4}(\tau)\right]\right\|_{\frac{10}{7}} \leq C\left\|D_{x}^{3 / 5} u(\tau)\right\|_{\frac{10}{3}}\|u(\tau)\|_{10}^{4} .
$$

Thus by (3.3), (3.9) and Young's inequality

$$
\begin{aligned}
(3.4) & \leq C\|\| D_{x}^{3 / 5} u\left\|_{L_{x}^{10 / 3}}\right\| u\left\|_{L_{x}^{10}}^{4}\right\|_{L^{2}[I]} \\
& \leq C\left\|D_{x}^{3 / 5} u\right\|_{L_{t \in I}^{10} L_{x}^{10 / 3}}\|u\|_{L_{x, t}^{10}[I]}^{4} .
\end{aligned}
$$

Consequently, from (3.3), (3.4), (3.5), (3.10), (3.1)

$$
\begin{aligned}
\left\|D_{x}^{3 / 5} u\right\|_{L_{t \in I}^{10} L_{x}^{10 / 3}} & \leq C\|u\|_{H^{1}}+C\|u\|_{L_{x, t}^{10}[I]}^{4}\left\|D_{x}^{3 / 5} u\right\|_{L_{t \in I}^{10} L_{x}^{10 / 5}} \\
& \leq C\|u\|_{H^{1}}+C \eta^{4}\left\|D_{x}^{3 / 5} u\right\|_{L_{t \in I}^{10} L_{x}^{10 / 3}}
\end{aligned}
$$

implying

$$
\left\|D_{x}^{3 / 5} u\right\|_{L_{t \in I}^{10} L_{x}^{10 / 3}} \leq C\|u\|_{H^{1}}
$$


Next, estimate again from (3.2)

$$
\begin{aligned}
\left\|u-e^{i(t-a) \Delta} u(a)\right\|_{L_{x, t}^{10}[I]} & \lesssim\left\|D_{x}^{3 / 5}\left[u-e^{i(t-a) \Delta} u(a)\right]\right\|_{L_{t \in I}^{10} L_{x}^{10 / 3}} \\
& =(3.4) \\
& \leq C \eta^{4}
\end{aligned}
$$

by $(3.10),(3.11),(3.1)$.

Thus, from (3.1), (3.12)

$$
\left\|e^{i(t-a) \Delta} u(a)\right\|_{L_{x, t}^{10}[I]} \sim \eta .
$$

Define next the Fourier restriction operators (wrt the $x$-variable)

$$
\begin{aligned}
P_{N} \phi & =\int_{|\xi| \leq N} \widehat{\phi}(\xi) e^{i x \cdot \xi} d \xi, \\
P^{N} \phi & =\int_{|\xi|>N} \widehat{\phi}(\xi) e^{i x \cdot \xi} d \xi, \\
\Delta_{N} \phi & =\int_{|\xi| \sim N} \widehat{\phi}(\xi) e^{i x \cdot \xi} d \xi .
\end{aligned}
$$

Since

$$
\begin{aligned}
\left\|P_{N}\left(e^{i(t-a) \Delta} u(a)\right)\right\|_{L_{x, t}^{10}[I]} & \leq\left[\int_{I}\left\|e^{i(t-a) \Delta} u(a)\right\|_{L_{x}^{6}}^{6}\left\|P_{N}\left(e^{i(t-a) \Delta} u(a)\right)\right\|_{\infty}^{4} d t\right]^{1 / 10} \\
& \lesssim\left(|I| N^{2}\right)^{1 / 10}\|u\|_{H^{1}}
\end{aligned}
$$

it follows from (3.13) that for

$$
N_{0} \sim\left(\frac{\eta}{\|u\|_{H^{1}}}\right)^{5}|I|^{-1 / 2}
$$

we have

$$
\left\|P^{N_{0}}\left(e^{i(t-a) \Delta} u(a)\right)\right\|_{L_{x, t}^{10}[I]} \sim \eta
$$

and hence, by the Littlewood-Paley theorem

$$
\left\|\left(\sum_{\substack{N>N_{0} \\ N \text { dyadic }}}\left|\Delta_{N}\left(e^{i(t-a) \Delta} u(a)\right)\right|^{2}\right)^{1 / 2}\right\|_{L_{x, t}^{10}[I]} \sim \eta
$$

or

$$
\sum_{\substack{N_{1} \geq N_{2} \geq N_{3} \geq N_{4} \geq N_{5} \geq N_{0} \\ \text { dyadic }}} \int_{I} d t \int d x \prod_{j=1}^{5}\left|\Delta_{N_{j}}\left(e^{i(t-a) \Delta} u(a)\right)\right|^{2} \geq \eta^{10} .
$$

Denote

$$
\sigma_{N}=\sup _{t \in I} N^{-1 / 2}\left\|\Delta_{N}\left(e^{i(t-a) \Delta} u(a)\right)\right\|_{\infty}
$$

(thus $\sigma_{N} \lesssim 1$ ) and estimate

$$
\begin{aligned}
\prod_{j=1}^{5} \mid \Delta_{N_{j}} & \left.\left(e^{i(t-a) \Delta} u(a)\right)\right|^{2} \leq\left|\Delta_{N_{1}}\left(e^{i(t-a) \Delta} u(a)\right)\right|^{2} \\
& \times\left|\Delta_{N_{2}}\left(e^{i(t-a) \Delta} u(a)\right)\right|^{\frac{4}{3}} N_{2}^{1 / 3} N_{3} N_{4} N_{5} \sigma_{N_{2}}^{2 / 3} \sigma_{N_{3}}^{2} \sigma_{N_{4}}^{2} \sigma_{N_{5}}^{2} .
\end{aligned}
$$


By Hölder's and Strichartz' inequalities

$$
\begin{aligned}
\int_{I} \int & d x\left|\Delta_{N_{1}}\left(e^{i(t-a) \Delta} u(a)\right)\right|^{2}\left|\Delta_{N_{2}}\left(e^{i(t-a) \Delta} u(a)\right)\right|^{\frac{4}{3}} \\
& \leq\left\|\Delta_{N_{1}}(\cdots)\right\|_{\frac{10}{3}}^{2}\left\|\Delta_{N_{2}}(\cdots)\right\|_{\frac{10}{3}}^{4 / 3} \leq C\left\|\Delta_{N_{1}} u(a)\right\|_{2}^{2}\left\|\Delta_{N_{2}} u(a)\right\|_{2}^{4 / 3} \\
& \leq C N_{1}^{-2} N_{2}^{-4 / 3}\left\|\Delta_{N_{1}} u(a)\right\|_{H^{1}}^{2} .
\end{aligned}
$$

Substitution of (3.17), (3.18) permits to bound the left member of (3.16) by

$$
\left[\sum_{\substack{N_{1} \geq \ldots \geq N_{5} \geq N_{0} \\ \text { dyadic }}}\left\|\Delta_{N_{1}} u(a)\right\|_{H^{1}}^{2} N_{1}^{-2} N_{2}^{-1} N_{3} N_{4} N_{5}\right]\left(\max _{N>N_{0}} \sigma_{N}^{20 / 3}\right) \leq C \max _{N>N_{0}} \sigma_{N}^{20 / 3}
$$

so that for some $N>N_{0}$

$$
\sigma_{N} \gtrsim \eta^{3 / 2}
$$

Consequently, there is some $t_{0} \in I$ and $x_{0} \in \mathbb{R}^{3}$ such that

$$
\left|\left(e^{i\left(t_{0}-a\right) \Delta} \Delta_{N} u(a)\left(x_{0}\right)\right)\right| \gtrsim \eta^{3 / 2} N^{1 / 2}
$$

where, by (3.15),

$$
N>C\left(\eta,\|u\|_{H^{1}}\right)|I|^{-1 / 2} .
$$

From (3.20), it follows easily that

$$
\left\|e^{i\left(t_{0}-a\right) \Delta} \Delta_{N} u(a)\right\|_{L^{2}\left[\left|x-x_{0}\right|<\frac{C}{N}\right]} \gtrsim N^{-1} \eta^{3 / 2}
$$

(where $\left.C=C\left(\eta,\|u\|_{H^{1}}\right)\right)$ and

$$
\left\|\nabla\left(e^{i\left(t_{0}-a\right) \Delta} u(a)\right)\right\|_{L^{2}\left[\left|x-x_{0}\right|<\frac{C}{N}\right]} \gtrsim \eta^{3 / 2} .
$$

Since $u$ is radial and

$$
\left\|\nabla\left(e^{i\left(t_{0}-a\right) \Delta} u(a)\right)\right\|_{2}=\|u(a)\|_{H^{1}}<C,
$$

(3.23) implies that

$$
\left|x_{0}\right|<C N^{-1}, C=C\left(\eta,\|u\|_{H^{1}}\right) .
$$

Observe that (3.22) remains valid for $\left|t-t_{0}\right|<c\left(\eta,\|u\|_{H^{1}}\right) N^{-2}$. Hence, by (3.24)

$$
\left\|e^{i(t-a) \Delta} \Delta_{N} u(a)\right\|_{L^{2}\left[|x|<\frac{C}{N}\right]} \gtrsim \eta^{3 / 2} N^{-1}
$$

and also

$$
\left\|\nabla\left(e^{i(t-a) \Delta} u(a)\right)\right\|_{L^{2}\left[|x|<\frac{C}{N}\right]} \gtrsim \eta^{3 / 2}
$$

for $\left|t-t_{0}\right|<C N^{-2}$ (the precise lower bound in (3.26) will be important later on). Similarly, from

$$
\left|\left(e^{i(t-a) \Delta} \Delta_{N} u(a)\right)\left(x_{0}\right)\right| \gtrsim \eta^{3 / 2} N^{1 / 2}
$$

for

$$
\left|t-t_{0}\right|<C\left(\eta,\|u\|_{H^{1}}\right) N^{-2}
$$

one deduces (taking (3.24) into account) that for $\left|t-t_{0}\right|<C N^{-2}$

$$
\left\|e^{i(t-a) \Delta} \Delta_{N} u(a)\right\|_{L^{6}\left[|x|<\frac{C}{N}\right]} \gtrsim \eta^{3 / 2}
$$


and

$$
\left\|e^{i(t-a) \Delta} u(a)\right\|_{L^{6}\left[|x|<\frac{C}{N}\right]} \gtrsim \eta^{3 / 2} .
$$

\section{Use of The MoraWetZ INEQUALITY}

Assume (1.1) is wellposed on a time interval $\mathcal{J}$ and

$$
\|u\|_{L_{x, t}^{10}[\mathcal{J}]}>M
$$

where $M$ is a large number, to be specified.

Let $\eta$ be as above and consider a sequence of times in $\mathcal{J}$

$$
a_{1}<a_{2}<\cdots<a_{J}
$$

such that

$$
\|u\|_{L_{x, t}^{10}\left[a_{j}, a_{j+1}\right]}=\eta
$$

Denote

$$
\mathcal{J}_{0}=\left[a_{1}, a_{J}\right]
$$

From the construction in section 3 , one gets then for each $j$ some $t_{j} \in\left[a_{j}, a_{j+1}\right]$ and $N_{j}>c\left(a_{j+1}-a_{j}\right)^{-1 / 2}$ such that (3.25), (3.26), (3.29) hold, i.e.

$$
\begin{aligned}
\left\|e^{i\left(t-a_{j}\right) \Delta} \Delta_{N_{j}} u\left(a_{j}\right)\right\|_{H^{1}} & \geq \eta^{3 / 2}, \\
\left\|\nabla\left(e^{i\left(t-a_{j}\right) \Delta} u\left(a_{j}\right)\right)\right\|_{L^{2}\left[|x|<C N_{j}^{-1}\right]} & \gtrsim \eta^{3 / 2},
\end{aligned}
$$

and

$$
\left\|e^{i\left(t-a_{j}\right) \Delta} u\left(a_{j}\right)\right\|_{L^{6}\left[|x|<C N_{j}^{-1}\right]} \gtrsim \eta^{3 / 2}
$$

for $\left|t-t_{j}\right|<c N_{j}^{-2}$.

From the integral equation

$$
u(t)=e^{i\left(t-a_{j}\right) \Delta} u\left(a_{j}\right)+i \int_{a_{j}}^{t} e^{i(t-\tau) \Delta}\left(u|u|^{4}\right)(\tau) d \tau
$$

it follows that for $t \in\left[a_{j}, a_{j+1}\right]$

$$
\begin{aligned}
\left\|u(t)-e^{i\left(t-a_{j}\right) \Delta} u\left(a_{j}\right)\right\|_{H^{1}} & \leq \int_{a_{j}}^{a_{j+1}} \int\left|e^{i \tau \Delta} \psi\right|\left|\left(D_{x} u\right)(\tau)\right||u(\tau)|^{4} d x d \tau \\
& \leq\left\|e^{i \tau \Delta} \psi\right\|_{L_{x, t}^{10 / 3}}\left\|D_{x} u\right\|_{L_{x, t}^{10 / 3}\left[a_{j}, a_{j+1}\right]}\|u\|_{L_{x, t}^{10}\left[a_{j}, a_{j+1}\right]}^{4} \\
& <C \eta^{4}
\end{aligned}
$$


by (4.2) and Strichartz' inequality. The bound on $\left\|D_{x} u\right\|_{L_{x, t}^{10 / 3}\left[a_{j}, a_{j+1}\right]}$ follows from the inequality (cf. section 2)

$$
\begin{aligned}
\left\|D_{x} u\right\|_{L_{x, t}^{10 / 3}\left[a_{j}, a_{j+1}\right]} & \leq\left\|D_{x} e^{i\left(t-a_{j}\right) \Delta} u\left(a_{j}\right)\right\|_{L_{x, t}^{10 / 3}} \\
& +\left\|\int_{a_{j}}^{a_{j+1}} \frac{1}{|t-\tau|^{3 / 5}}\right\|\left|D_{x} u\right|(\tau)|u(\tau)|^{4}\left\|_{\frac{10}{7}}\right\|_{L_{t}^{10 / 3}} \\
& \leq C\left\|D_{x} u\left(a_{j}\right)\right\|_{2}+\left\|\left|D_{x} u\right||u|^{4}\right\|_{L_{x, t}^{10 / 7}\left[a_{j}, a_{j+1}\right]} \\
& \leq C\|u\|_{H^{1}}+C\left\|D_{x} u\right\|_{L_{x, t}^{10 / 3}\left[a_{j}, a_{j+1}\right]}\|u\|_{L_{x, t}^{10}\left[a_{j}, a_{j+1}\right]}^{4} \\
& \leq C\|u\|_{H^{1}}+C \eta^{4}\left\|D_{x} u\right\|_{L_{x, t}^{10 / 3}\left[a_{j}, a_{j+1}\right]} .
\end{aligned}
$$

From (4.6), we get also

$$
\left\|u(t)-e^{i\left(t-a_{j}\right) \Delta} u\left(a_{j}\right)\right\|_{6}<C \eta^{4} .
$$

Hence, by (4.3)-(4.5), it follows that for

$$
\left.t \in\left[a_{j}, a_{j+1}\right], \mid t-t_{j}\right]<c N_{j}^{-2}
$$

we have

$$
\begin{aligned}
\left\|\Delta_{N_{j}} u(t)\right\|_{H^{1}} & \geq \eta^{3 / 2}, \\
\|\nabla u(t)\|_{L^{2}\left[|x|<C N_{j}^{-1]}\right.} & \gtrsim \eta^{3 / 2}, \\
\|u(t)\|_{L^{6}\left[|x|<C N_{j}^{-1}\right]} & \gtrsim \eta^{3 / 2} .
\end{aligned}
$$

Fix a small number $\kappa>0$ and assume that

$$
N_{j}^{-2}<\kappa\left(a_{J}-a_{1}\right)=\kappa\left|\mathcal{J}_{0}\right| \text { for each } j .
$$

Recalling inequality (2.2) applied to the time interval $\mathcal{J}_{0}=\left[a_{1}, a_{J}\right]$, we get

$$
\begin{aligned}
C\left(a_{J}-a_{1}\right)^{1 / 2} & >\int_{a_{1}}^{a_{J}} d t \int d x \frac{|u(x, t)|^{6}}{|x|} d x d t \\
& >\sum_{j=1}^{J-1} \int_{a_{j}}^{a_{j+1}} d t\left[C^{-1} N_{j}\|u(t)\|_{L^{6}\left[|x|<C N_{j}^{-1}\right]}^{6}\right] .
\end{aligned}
$$

Restricting $t \in\left[a_{j}, a_{j+1}\right]$ to $\left|t-t_{j}\right|<c N_{j}^{-2}<a_{j+1}-a_{j}$, (4.11), (4.12) imply

$$
(4.13)>c(\eta) \sum_{j=1}^{J-1} N_{j}^{-2} \kappa^{-1 / 2}\left(a_{J}-a_{1}\right)^{-1 / 2} .
$$

Hence

$$
\sum_{j=1}^{J-1} N_{j}^{-2}<C(\eta) \kappa^{1 / 2}\left(a_{J}-a_{1}\right)<\kappa^{1 / 3} \sum_{j=1}^{J-1}\left(a_{j+1}-a_{j}\right)
$$

taking

$$
\kappa<C(\eta)
$$

Thus (4.15) implies the existence of some interval

$$
I=\left[a_{j}, a_{j+1}\right] \subset \mathcal{J}_{0}
$$


for which

$$
N_{j}^{-2}<\kappa^{1 / 3}|I| .
$$

This is the setup required for the continuation of the argument.

The conclusion is subject to hypothesis (4.12). Assume otherwise there is $j_{1}$ such that

$$
N_{j_{1}}^{-2}>\kappa\left(a_{J}-a_{1}\right) .
$$

Consider then either the sequence

$$
a_{1}<a_{2}<\cdots<a_{j_{1}-1}, \mathcal{J}_{1}=\left[a_{1}, a_{j_{1}-1}\right]
$$

or

$$
a_{j_{1}+1}<\cdots<a_{J}, \mathcal{J}_{1}=\left[a_{j_{1}+1}, a_{J}\right]
$$

such that $J_{1}=j_{1}$ (or $J-j_{1}$ ) is at least $J / 2$ and repeat the preceding. Remark that since (4.17) implies that $a_{j+1}-a_{j}>c \kappa\left(a_{J}-a_{1}\right)$, necessarily

$$
\left|\mathcal{J}_{1}\right|<(1-c \kappa)\left|\mathcal{J}_{0}\right| \text {. }
$$

Let $r$ be an integer (in particular depending on $\kappa$ ) and suppose (4.12) fails for $r$ repetitions (which we may perform provided $\log J \gg r$, hence for $M$ in (4.1) sufficiently large).

Thus we get indices

$$
j_{1}, j_{2}, \ldots, j_{r}
$$

times

$$
t_{j_{1}}, t_{j_{2}}, \ldots, t_{j_{r}}
$$

and numbers

$$
N_{j_{1}}, N_{j_{2}}, \ldots, N_{j_{r}}
$$

satisfying

$$
\begin{aligned}
t_{j_{s}} \in\left[a_{j_{s}}, a_{j_{s+1}}\right] & \subset \mathcal{J}_{s-1}, \\
C\left|\mathcal{J}_{s-1}\right| \geq C\left|a_{j_{s}+1}-a_{j_{s}}\right|>N_{j_{s}}^{-2} & >\kappa\left|\mathcal{J}_{s-1}\right|, \\
\left\|\Delta_{N_{j_{s}}} u\left(t_{j_{s}}\right)\right\|_{H^{1}} & \gtrsim \eta^{3 / 2}(\text { from (4.9)). }
\end{aligned}
$$

Our next purpose is to show that when $r=r(\kappa)$ is taken sufficiently large, the boundedness

$$
\sup _{t}\|u(t)\|_{H^{1}}<\infty
$$

will be contradicted.

It follows from (4.21) that for all $N<N_{j_{s}}$

$$
\left\|P^{N} u\left(t_{j_{s}}\right)\right\|_{2}>c(\eta) N_{j_{s}}^{-1} .
$$

From equation (1.1), we get

$$
\begin{aligned}
\frac{d}{d t}\left\|P^{N} u(t)\right\|_{2}^{2} & =\operatorname{Im}\left\langle P^{N} u(t), P^{N}\left[u(t)|u(t)|^{4}\right]\right\rangle \\
& =\operatorname{Im}\left\langle P^{N} u(t), u(t)|u(t)|^{4}-P^{N} u(t)\left|P^{N} u(t)\right|^{4}\right\rangle
\end{aligned}
$$

which, writing $u(t)=P_{N} u(t)+P^{N} u(t)$, may by Hölder's inequality be bounded by

$$
\left\|\left[P^{N} u(t)\right]\left[P_{N} u(t)\right]\right\|_{L_{x}^{3}}\|u(t)\|_{L_{x}^{6}}^{4} .
$$


Hence, for $N=N_{s}<N_{j_{s}}$, by (4.22)

$$
\begin{aligned}
\left\|P^{N_{s}} u\left(t_{j_{r}}\right)\right\|_{2}^{2} & \geq\left\|P^{N_{s}} u\left(t_{j_{s}}\right)\right\|_{2}^{2}-C \int_{t_{j_{s}}}^{t_{j_{r}}}\left\|P^{N_{s}} u(t) \cdot P_{N_{s}} u(t)\right\|_{3}|d t| \\
& >c N_{j_{s}}^{-2}-C \int_{t_{j_{s}}}^{t_{j_{r}}}\left\|P^{N_{s}} u(t) \cdot P_{N_{s}} u(t)\right\|_{3}|d t| .
\end{aligned}
$$

Observe that the integral term in (4.24) is certainly bounded by $C\left|t_{j_{s}}-t_{j_{r}}\right|<$ $C\left|\mathcal{J}_{s-1}\right|<C \kappa^{-1} N_{j_{s}}^{-2}$, from (4.19), (4.20), and our aim is to pick $N_{s}$ such that it becomes $o\left(N_{j_{s}}^{-2}\right)$.

To do this, observe that if we let

$$
M_{\alpha}=2^{-\alpha} N_{j_{s}} \quad(\alpha=0,1,2, \ldots),
$$

then

$$
\begin{aligned}
\sum_{\alpha=1}^{\beta}\left\|P^{M_{\alpha}} \psi \cdot P_{M_{\alpha}} \psi\right\|_{3} & \leq \sum_{\alpha=1}^{\beta} \sum_{\substack{L_{1}>M_{\alpha}>L_{2} \\
\text { dyadic }}}\left\|\Delta_{L_{1}} \psi \cdot \Delta_{L_{2}} \psi\right\|_{3} \\
& \leq \sum_{\alpha=1}^{\beta} \sum_{L_{1}>M_{\alpha}>L_{2}}\left\|\Delta_{L_{1}} \psi\right\|_{3}\left\|\Delta_{L_{2}} \psi\right\|_{\infty} \\
& \leq C \sum_{\alpha=1}^{\beta} \sum_{L_{1}>M_{\alpha}>L_{2}}\left(\frac{L_{2}}{L_{1}}\right)^{1 / 2}\left\|\Delta_{L_{1}} \psi\right\|_{H^{1}}\left\|\Delta_{L_{2}} \psi\right\|_{H^{1}} \\
& <\sum_{L_{1}>L_{2}}\left(\frac{L_{2}}{L_{1}}\right)^{1 / 2-}\left\|\Delta_{L_{1}} \psi\right\|_{H^{1}}\left\|\Delta_{L_{2}} \psi\right\|_{H^{1}} \\
& <C\|\psi\|_{H^{1}}^{2} .
\end{aligned}
$$

Hence, letting $\psi=u(t)$, inequality (4.25) gives some

$$
2^{-1 / \kappa^{2}} N_{j_{s}}<N_{s}<N_{j_{s}}
$$

such that

$$
\int_{t_{j_{s}}}^{t_{j_{r}}}\left\|P^{N_{s}} u(t) \cdot P_{N_{s}} u(t)\right\|_{3}|d t|<C \kappa^{2}\left|t_{j_{s}}-t_{j_{r}}\right|<C \kappa N_{j_{s}}^{-2} .
$$

For this choice of $N_{s},(4.24),(4.27),(4.26)$ imply

$$
\begin{aligned}
N_{j_{s}}^{2}\left\|P^{N_{s}} u\left(t_{j_{r}}\right)\right\|_{2}^{2} & >c, \\
N_{s}^{2}\left\|P^{N_{s}} u\left(t_{j_{r}}\right)\right\|_{2}^{2} & >c 4^{-1 / \kappa^{2}} .
\end{aligned}
$$

By (4.20), (4.26)

$$
\begin{aligned}
c\left|\mathcal{J}_{s-1}\right|^{-1 / 2} & <N_{j_{s}}<\kappa^{-1 / 2}\left|\mathcal{J}_{s-1}\right|^{-1 / 2}, \\
c 2^{-1 / \kappa^{2}}\left|\mathcal{J}_{s-1}\right|^{-1 / 2} & <N_{s}<\kappa^{-1 / 2}\left|\mathcal{J}_{s-1}\right|^{-1 / 2}
\end{aligned}
$$

where

$$
\begin{aligned}
& \mathcal{J}_{0} \supset \mathcal{J}_{1} \supset \cdots \supset \mathcal{J}_{s-1} \supset \mathcal{J}_{s} \supset \cdots, \\
& \left|\mathcal{J}_{s}\right|<(1-C \kappa)\left|\mathcal{J}_{s-1}\right| .
\end{aligned}
$$


Hence, as a consequence of (4.29), (4.30)

$$
\sum\left\{N_{s}^{2} \mid N_{s}<N\right\}<C \kappa^{-2} 4^{1 / \kappa^{2}} N^{2} .
$$

Summation of (4.28) for $s=1, \ldots, r$ gives then by (4.31)

$$
\begin{aligned}
c r 4^{-1 / \kappa^{2}}<\sum_{s=1}^{r} N_{s}^{2}\left\|P^{N_{s}} u\left(t_{j_{r}}\right)\right\|_{2}^{2} & =\sum_{s=1}^{r} N_{s}^{2} \sum_{\substack{N \geq N_{s} \\
N \text { dyadic }}}\left\|\Delta_{N} u\left(t_{j_{r}}\right)\right\|_{2}^{2} \\
& \leq C \kappa^{-2} 4^{1 / \kappa^{2}} \sum N^{2}\left\|\Delta_{N} u\left(t_{j_{r}}\right)\right\|_{2}^{2} \\
& =C \kappa^{-2} 4^{1 / \kappa^{2}}\left\|u\left(t_{j_{r}}\right)\right\|_{H^{1}}^{2}
\end{aligned}
$$

leading to the desired contradiction for $r>r(\kappa)$.

Hence, the hypothesis (4.12) needs to hold on one of the intervals $\mathcal{J}_{0} \supset \mathcal{J}_{1} \supset$ $\cdots \supset \mathcal{J}_{r}$ and one may thus claim (4.16) for some $j$.

\section{USE OF THE PSEUdO-CONFORMAL CONSERVATION LAW}

Our starting point is the situation (4.16), thus a time-interval $I=[a, b], t_{0} \in I$ and $N$ such that

$$
\begin{aligned}
N^{-2} & <\kappa^{1 / 3}|I|, \\
\|u\|_{L_{x, t}^{10}[I]} & =\eta,
\end{aligned}
$$

and, cf. (4.10),

$$
\left\|\nabla u\left(t_{0}\right)\right\|_{L^{2}\left[|x|<C(\eta) N^{-1}\right]} \gtrsim \eta^{3 / 2} .
$$

More precisely, assuming $\mathcal{J}$ is a time interval on which (1.1) is wellposed and

$$
\|u\|_{L_{x, t}^{10}[\mathcal{J}]}>M
$$

one may write $\mathcal{J}$ as a union of 3 consecutive intervals

$$
\mathcal{J}=\mathcal{J}_{-} \cup \mathcal{J}_{0} \cup \mathcal{J}_{+},
$$

each satisfying

$$
\|u\|_{L_{x, t}^{10}\left[\mathcal{J}_{-, 0,+}\right]}>\frac{M}{3},
$$

and perform the construction from section 4 in the middle interval $\mathcal{J}_{0}$, thus $I \subset \mathcal{J}_{0}$. We then distinguish 2 cases.

(i) $t_{0}-a<b-t_{0}$.

In this section, going forward in time, we will aim to disprove

$$
\|u\|_{L_{x, t}^{10}\left[\mathcal{J}_{+}\right]}>\frac{M}{3} .
$$

(ii) $\underline{b-t_{0}<t_{0}-a}$.

Then, going backwards in time, we aim to disprove that

$$
\|u\|_{L_{x, t}^{10}\left[\mathcal{J}_{-}\right]}>\frac{M}{3} .
$$

Our reasoning will involve an inductive argument on the size of the Hamiltonian. We assume, say, that for an IVP

$$
\left\{\begin{array}{l}
i W_{t}+\Delta W-W|W|^{4}=0, \\
W(0)=\psi \in H^{1} \quad(\psi \text { radial and smooth }),
\end{array}\right.
$$


such that

$$
H(\psi)<H(u)-\eta^{4},
$$

there is global wellposedness and a uniform estimate

$$
\|W\|_{L_{x, t}^{10}[S]}<M_{1},
$$

for any unit-time interval $S$ ((5.8) implies global wellposedness). Recall that there is global wellposedness of $(5.6)$ and $\|W\|_{L_{x, t}^{10}(\mathbb{R})}<\infty$ provided $H(\psi)$ is sufficiently small. Thus our purpose is to show that under assumption (5.6)-(5.8) the hypothesis (5.4) with $M$ sufficiently large leads to a contradiction.

We assume case (i); the other case is similar.

Consider the IVP

$$
\left\{\begin{array}{l}
i u_{t}+\Delta u-u|u|^{4}=0, \\
\left.u\right|_{t=t_{0}}=u\left(t_{0}\right)
\end{array}\right.
$$

By construction

$$
\|u\|_{L^{10}\left[t_{0}, b\right]} \leq \eta
$$

and $N$ in (5.3) fulfills

$$
N^{-2}<2 \kappa^{1 / 3}\left(b-t_{0}\right)
$$

From (5.3), we derive the following.

Lemma 5.12. There is a radial bump function $\zeta$ such that

$$
\left\{\begin{array}{l}
\zeta=1 \text { for }|x|<C(\eta) N^{-1}, \\
\zeta=0 \text { for }|x|>C^{\prime}(\eta) N^{-1},
\end{array}\right.
$$

and

$$
\left\|u\left(t_{0}\right)(1-\zeta)\right\|_{H^{1}}^{2}<\left\|u\left(t_{0}\right)\right\|_{H^{1}}^{2}-c \eta^{3} .
$$

Proof. We construct $\zeta$ satisfying (5.13) and such that

$$
\left\|u\left(t_{0}\right) \nabla \zeta\right\|_{2}<\eta^{4}
$$

It then follows from (5.3) that

$$
\begin{aligned}
\left\|u\left(t_{0}\right)(1-\zeta)\right\|_{H^{1}}^{2} & \leq\left\|\nabla u\left(t_{0}\right)\right\|_{L^{2}\left[|x|>C(\eta) N^{-1}\right]}^{2}+C \eta^{4} \\
& \leq\left\|\nabla u\left(t_{0}\right)\right\|_{2}^{2}-\left\|\nabla u\left(t_{0}\right)\right\|_{L^{2}\left[|x|<C(\eta) N^{-1}\right]}^{2}+C \eta^{4} \\
& <\left\|\nabla u\left(t_{0}\right)\right\|_{2}^{2}-c \eta^{3}+C \eta^{4} \\
& <\left\|u\left(t_{0}\right)\right\|_{H^{1}}^{2}-c \eta^{3} .
\end{aligned}
$$

To get (5.15), consider smooth bump functions $\zeta_{0}, \zeta_{1}, \ldots$ satisfying

$$
\left\{\begin{array}{l}
\zeta_{s}=1 \text { for }|x|<2^{s} C(\eta) N^{-1} \\
\zeta_{s}=0 \text { for }|x|>2^{s+1} C(\eta) N^{-1} \\
\left|D^{(\ell)} \zeta_{s}\right| \lesssim\left(2^{-s} N\right)^{\ell}(\ell=1,2) .
\end{array}\right.
$$


Then, by (5.16) and Hölder's inequality

$$
\begin{aligned}
& \sum_{s=1}^{r} \| \\
& \quad \leq C\left(t_{0}\right) \nabla \zeta_{s}\left\|_{2}^{6} \leq C(\eta) \sum_{s=1}^{r}\left(2^{-s} N\right)^{6}\right\| u\left(t_{0}\right)\left\|_{L^{2}\left[2^{s} C(\eta) N^{-1}<|x|<2^{s+1} C(\eta) N^{-1}\right]}^{r}\left(2^{-s} N\right)^{6}\left(2^{s+1} N^{-1}\right)^{6}\right\| u\left(t_{0}\right) \|_{L^{6}\left[2^{s} C(\eta) N^{-1}<|x|<2^{s+1} C(\eta) N^{-1}\right]}^{6} \\
& \quad \leq C(\eta)\left\|u\left(t_{0}\right)\right\|_{6}^{6} \\
& \quad \leq C(\eta)\left\|u\left(t_{0}\right)\right\|_{H^{1}}^{6}
\end{aligned}
$$

and hence there is some $s \lesssim C(\eta)$ for which

$$
\left\|u\left(t_{0}\right) \nabla \zeta_{s}\right\|_{2}<\eta^{4}
$$

This proves the lemma.

Consider next the IVP

$$
\left\{\begin{array}{l}
i v_{t}+\Delta v-v|v|^{4}=0 \\
\left.v\right|_{t=t_{0}}=\zeta u\left(t_{0}\right)
\end{array}\right.
$$

\section{Lemma 5.19.}

$$
\left\|e^{i\left(t-t_{0}\right) \Delta} v\left(t_{0}\right)\right\|_{L_{x, t}^{10}\left[t_{0}, b\right]} \leq C \eta
$$

Proof. First, the integral equation

$$
u(t)=e^{i\left(t-t_{0}\right) \Delta} u\left(t_{0}\right)+i \int_{t_{0}}^{t} e^{i(t-\tau) \Delta}\left(u|u|^{4}\right)(\tau) d \tau
$$

implies (cf. section 3)

$$
\left\|e^{i\left(t-t_{0}\right) \Delta} u\left(t_{0}\right)\right\|_{L_{x, t}^{10}[I]} \leq\|u\|_{L_{x, t}^{10}[I]}+C\left\|D_{x}^{3 / 5} u\right\|_{L_{t \in I}^{10} L_{x}^{10 / 3}}\|u\|_{L_{x, t}^{10}[I]}^{4}<2 \eta
$$

where $I=\left[t_{0}, b\right]$.

Next, since $\widehat{v\left(t_{0}\right)}=\widehat{\zeta} * \widehat{u\left(t_{0}\right)}$ and $\|\widehat{\zeta}\|_{1}<C$, we get

$$
\begin{aligned}
\left|e^{i\left(t-t_{0}\right) \Delta} v\left(t_{0}\right)\right| & =\left|\int \widehat{v\left(t_{0}\right)}(\xi) e^{i\left(x \cdot \xi+\left(t-t_{0}\right) \xi^{2}\right)} d \xi\right| \\
& \leq \int\left|\int \widehat{u\left(t_{0}\right)}\left(\xi-\xi_{1}\right) e^{i\left(x \cdot \xi+\left(t-t_{0}\right) \xi^{2}\right)} d \xi\right| \widehat{\zeta}\left(\xi_{1}\right) \mid d \xi_{1}, \\
\left\|e^{i\left(t-t_{0}\right) \Delta} v\left(t_{0}\right)\right\|_{L_{x, t}^{10}[I]} & \lesssim \sup _{\xi_{1}}\left\|\int \widehat{u\left(t_{0}\right)}\left(\xi-\xi_{1}\right) e^{i\left(x \xi+\left(t-t_{0}\right) \xi^{2}\right)} d \xi\right\|_{L_{x, t}^{10}[I]}
\end{aligned}
$$

where, by a change of variable,

$$
\begin{aligned}
\left|\int \widehat{u\left(t_{0}\right)}\left(\xi-\xi_{1}\right) e^{i\left(\left(x \cdot \xi+\left(t-t_{0}\right) \xi^{2}\right)\right.} d \xi\right| & =\left|\int \widehat{u}\left(t_{0}\right)(\xi) e^{i\left(\left(x+2\left(t-t_{0}\right) \xi_{1}\right) \cdot \xi+\left(t-t_{0}\right) \xi^{2}\right)} d \xi\right| \\
& =\left|\left(e^{i\left(t-t_{0}\right) \Delta} u\left(t_{0}\right)\right)\left(x+2\left(t-t_{0}\right) \xi_{1}\right)\right| .
\end{aligned}
$$

(5.19) then follows from (5.20), (5.21), (5.22).

Since

$$
v(t)=e^{i\left(t-t_{0}\right) \Delta} v\left(t_{0}\right)+i \int_{t_{0}}^{t} e^{i(t-\tau) \Delta}\left(v|v|^{4}\right)(\tau) d \tau
$$


it follows again from the estimates in section 3 that

$$
\begin{aligned}
\left\|D_{x}^{3 / 5} v\right\|_{L_{t \in I}^{10} L_{x}^{10 / 3}} & \lesssim\left\|v\left(t_{0}\right)\right\|_{H^{1}}+\left\|D_{x}^{3 / 5} v\right\|_{L_{t \in I}^{10} L_{x}^{10 / 3}}\|v\|_{L_{x, t}^{10}[I]}^{4}, \\
\|v\|_{L_{x, t}^{10}[I]} & \lesssim\left\|e^{i\left(t-t_{0}\right) \Delta} v\left(t_{0}\right)\right\|_{L_{x, t}^{10}[I]}+\left\|D_{x}^{3 / 5} v\right\|_{L_{t \in I}^{10} L_{x}^{10 / 3}}^{10}\|v\|_{L_{x, t}^{10}[I]}^{4} \\
& \stackrel{\text { by }(5.19)}{\lesssim} \eta+\left\|D_{x}^{3 / 5} v\right\|_{L_{t \in I}^{10} L_{x}^{10 / 3}}\|v\|_{L_{x, t}^{10}[I]}^{4}
\end{aligned}
$$

which permits us to conclude that

$$
\left\|D_{x}^{3 / 5} v\right\|_{L_{t \in I}^{10} L_{x}^{10 / 3}}<C,\|v\|_{L_{x, t}^{10}[I]} \lesssim \eta .
$$

Thus in particular (5.18) is wellposed on $I=\left[t_{0}, b\right]$.

Recall next the pseudo-conformal conservation law (cf. [G-V2], [Caz])

$$
\begin{aligned}
\|(x+ & \left.2 i\left(t-t_{0}\right) \nabla\right) v(t)\left\|_{2}^{2}+\frac{4}{3}\left(t-t_{0}\right)^{2}\right\| v(t) \|_{6}^{6} \\
& =\left\|x v\left(t_{0}\right)\right\|_{2}^{2}-\frac{16}{3} \int_{t_{0}}^{t} s \int_{\mathbb{R}^{3}}|v(s, x)|^{6} d x d s \\
& \leq\left\|x v\left(t_{0}\right)\right\|_{2}^{2}
\end{aligned}
$$

valid on $I$ and any larger interval $\left[t_{0}, b^{\prime}\right], b^{\prime}>b$ on which (5.18) is wellposed. Since by (5.18) and (5.13)

$$
\left\||x| v\left(t_{0}\right)\right\|_{2}^{2}=\left\||x| \zeta u\left(t_{0}\right)\right\|_{2}^{2} \leq C|||x| \zeta \|_{3}^{2}<C(\eta) N^{-4},
$$

we deduce from (5.27) that

$$
\|v(t)\|_{L_{x}^{6}}^{6} \leq C(\eta) \frac{1}{\left(\left|t-t_{0}\right| N^{2}\right)^{2}} .
$$

Hence, by (5.11)

$$
\|v(t)\|_{6}^{6} \leq C(\eta) \kappa^{2 / 3} \text { for } t>b .
$$

It follows from (5.30) that (5.18) remains wellposed on $[b, \infty[$ and

$$
\|v\|_{L_{x, t}^{10}[b, \infty[}<\kappa^{1 / 15} \text {. }
$$

Indeed, if (5.31) fails, we may find $b_{1}>b$ such that

$$
\|v\|_{L_{x, t}^{10}\left[b, b_{1}\right]}=\kappa^{1 / 15}
$$

and, replacing $u$ by $v$ and $\eta$ by $\kappa^{1 / 15}$ in assumption (3.1), (4.11) gives some $t \in\left[b, b_{1}\right]$ such that in particular

$$
\|v(t)\|_{6} \gtrsim \kappa^{1 / 10}
$$

contradicting (5.30). Thus (5.31) holds.

From (5.31), we get also

$$
\left\|D_{x}^{3 / 5} v\right\|_{L_{t \in[b, \infty}^{10} L_{x}^{10 / 3}}<C \text { and }\left\|D_{x} v\right\|_{L_{x, t}^{10 / 3}[b, \infty]}<C .
$$

Denote

$$
w=u-v
$$

satisfying the difference equation

$$
\left\{\begin{array}{l}
i w_{t}+\Delta w-u|u|^{4}+(u-w)|u-w|^{4}=0 \\
w\left(t_{0}\right)=(1-\zeta) u\left(t_{0}\right) .
\end{array}\right.
$$


From (5.10), (5.26), (5.34)

$$
\|w\|_{L_{x, t}^{10}[I]} \lesssim \eta,\left\|D_{x}^{3 / 5} w\right\|_{L_{t \in I}^{10} L_{x}^{10 / 3}}<C,\left\|D_{x} w\right\|_{L_{x, t}^{10 / 3}[I]}<C .
$$

From the integral equation

$$
w(t)=e^{i\left(t-t_{0}\right) \Delta} w\left(t_{0}\right)+i \int_{t_{0}}^{t} e^{i(t-\tau) \Delta}\left[u|u|^{4}-(u-w)|u-w|^{4}\right](\tau) d \tau
$$

it follows that for $t \in I$

$$
\begin{aligned}
\| w(t) & -e^{i\left(t-t_{0}\right) \Delta} w\left(t_{0}\right)\left\|_{H^{1}} \lesssim\right\| D_{x}\left[u|u|^{4}-(u-w)|u-w|^{4}\right] \|_{L_{x, t}^{10 / 7}[I]} \\
& \lesssim\left(\left\|D_{x} u\right\|_{L^{10 / 3}[I]}+\left\|D_{x} w\right\|_{L^{10 / 3}[I]}\right)\left(\|u\|_{L^{10}[I]}+\|w\|_{L^{10}[I]}\right)^{4} \\
& \lesssim \eta^{4}
\end{aligned}
$$

Thus, from (5.37), (5.14)

$$
\|w(b)\|_{H^{1}}^{2} \leq\left\|w\left(t_{0}\right)\right\|_{H^{1}}^{2}+C \eta^{4}=\left\|(1-\zeta) u\left(t_{0}\right)\right\|_{H^{1}}^{2}+C \eta^{4}<\left\|u\left(t_{0}\right)\right\|_{H^{1}}^{2}-c \eta^{3} .
$$

Also

$$
\|w(b)\|_{6}^{6}-\left\|w\left(t_{0}\right)\right\|_{6}^{6}=3 \int_{t_{0}}^{b} d t \int d x \operatorname{Re}\left[|w|^{4} \bar{w} \dot{w}\right]
$$

which, by $(5.35),(5.36)$ is bounded by

$$
\begin{aligned}
\int_{I} d t & \int d x\left[|\nabla w|^{2}|w|^{4}+|w|^{5}\left(|u|^{5}+|w|^{5}\right)\right] \\
& \lesssim\left\|D_{x} w\right\|_{L_{x, t}^{10 / 3}[I]}^{2}\|w\|_{L^{10}[I]}^{4}+\|w\|_{L^{10}[I]}^{5}\left(\|u\|_{L^{10}[I]}^{5}+\|w\|_{L^{10}[I]}^{5}\right) \\
& \lesssim \eta^{4} .
\end{aligned}
$$

Hence, from (5.38), (5.39)

$$
\begin{aligned}
H(w(b)) & =\frac{1}{2}\|w(b)\|_{H^{1}}^{2}+\frac{1}{6}\|w(b)\|_{6}^{6} \\
& <\frac{1}{2}\left\|u\left(t_{0}\right)\right\|_{H^{1}}^{2}-c \eta^{3}+\frac{1}{6}\left\|w\left(t_{0}\right)\right\|_{6}^{6}+C \eta^{4} \\
& \leq \frac{1}{2}\left\|u\left(t_{0}\right)\right\|_{H^{1}}^{2}+\frac{1}{6}\left\|u\left(t_{0}\right)\right\|_{6}^{6}-c \eta^{3} \\
& =H\left(u\left(t_{0}\right)\right)-c \eta^{3}
\end{aligned}
$$

and the initial data $\psi=w(b)$ satisfies thus assumption (5.7).

From the inductive assumption discussed in the beginning of this section, it follows that the IVP

$$
\left\{\begin{array}{l}
i W_{t}+\Delta W-W|W|^{4}=0, \\
W(b)=w(b)
\end{array}\right.
$$

is globally wellposed and $W$ satisfies (5.8). In particular

$$
\|W\|_{L_{x, t}^{10}([b, \infty[\cap \mathcal{J})}<M_{1}
$$

On the other hand, by (5.5), (5.31), we have that

$$
\frac{M}{3}-\kappa^{1 / 15}<\|w\|_{L^{10}\left[\mathcal{J}_{+}\right]} \leq\|w\|_{L^{10}([b, \infty[\cap \mathcal{J})}
$$

and we are going to show that (5.42), (5.43) are contradictory. 
Denote

$$
\Gamma=w-W
$$

hence

$$
u=v+W+\Gamma
$$

satisfying by (5.9), (5.18), (5.41) the equation

$$
\left\{\begin{array}{l}
i \Gamma_{t}+\Delta \Gamma-(v+W+\Gamma)|v+W+\Gamma|^{4}+v|v|^{4}+W|W|^{4}=0 \\
\Gamma(b)=0
\end{array}\right.
$$

Our aim is to show that $\Gamma$ remains in fact small. If $v$ were $0,(5.45)$ would imply indeed $\Gamma=0$. Now $v$ fulfills (5.31) where $\kappa$ may be assumed arbitrarily small wrt $M_{1}$ (choosing $M$ large enough, according to the argument in section 4).

Denote the interval

$$
\left[b, \infty\left[\cap \mathcal{J}=\left[b, b^{\prime}\right]=\mathcal{K} .\right.\right.
$$

In order to perform our perturbative analysis, notice that also, by interpolation

$$
\left\|D_{x}^{3 / 5} v\right\|_{L_{t \in \mathcal{K}}^{10} L_{x}^{10 / 3}} \leq\|v\|_{L_{x, t}^{10}[\mathcal{K}]}^{1 / 7}\left\|D_{x}^{7 / 10} v\right\|_{L_{t \in \mathcal{K}}^{10} L_{x}^{3}}^{6 / 7}
$$

where, by (5.33)

$$
\begin{aligned}
\left\|D_{x}^{7 / 10} v\right\|_{L_{t \in \mathcal{K}}^{10} L_{x}^{3}} & \leq\left\|D_{x}^{11 / 20} v\right\|_{L_{t \in \mathcal{K}}^{\infty} L_{x}^{20 / 7}}^{2 / 3}\left\|D_{x} v\right\|_{L_{x, t}^{10 / 3}[\mathcal{K}]}^{1 / 3} \\
& \leq\left\|D_{x} v\right\|_{L_{t \in \mathcal{K}}^{\infty} L_{x}^{2}}^{2 / 3}\left\|D_{x} v\right\|_{L^{10 / 3}[\mathcal{K}]}^{1 / 3}<C .
\end{aligned}
$$

Hence, (5.31), (5.47), (5.48) imply that

$$
\left\|D_{x}^{3 / 5} v\right\|_{L_{t \in \mathcal{K}}^{10} L_{x}^{10 / 3}}<\kappa^{1 / 120} .
$$

Assuming $I=\left[b_{1}, b_{2}\right] \subset \mathcal{K}$, the integral equation for $t \in I$

$$
\begin{aligned}
\Gamma(t)= & e^{i\left(t-b_{1}\right) \Delta} \Gamma\left(b_{1}\right) \\
& +i \int_{b_{1}}^{t} e^{i(t-\tau) \Delta}\left[(v+W+\Gamma)|v+W+\Gamma|^{4}-v|v|^{4}-W|W|^{4}\right](\tau) d \tau
\end{aligned}
$$

implies that

$$
\begin{aligned}
& \left\|D_{x}^{3 / 5} \Gamma\right\|_{L_{t \in I}^{10} L_{x}^{10 / 3}} \leq\left\|D_{x}^{3 / 5}\left(e^{i\left(t-b_{1}\right) \Delta} \Gamma\left(b_{1}\right)\right)\right\|_{L_{t \in I}^{10} L_{x}^{10 / 3}} \\
& +\left\|D_{x}^{3 / 5} v\right\|_{L_{t \in I}^{10} L_{x}^{10 / 3}}\left(\|v\|_{L^{10}[I]}+\|W\|_{L^{10}[I]}\right)^{4} \\
& +\left\|D_{x}^{3 / 5} W\right\|_{L_{t \in I}^{10} L_{x}^{10 / 3}}\|v\|_{L^{10}[I]}\left(\|v\|_{L^{10}[I]}+\|W\|_{L^{10}[I]}\right)^{3} \\
& +\left\|D_{x}^{3 / 5} \Gamma\right\|_{L_{t \in I}^{10} L_{x}^{10 / 3}}\left(\|v\|_{L^{10}[I]}+\|W\|_{L^{10}[I]}+\|\Gamma\|_{L^{10}[I]}\right)^{4} \\
& +\|\Gamma\|_{L^{10}[I]}\left(\left\|D_{x}^{3 / 5} v\right\|_{L_{t \in I}^{10} L_{x}^{10 / 3}}+\left\|D_{x}^{3 / 5} W\right\|_{L_{t \in I}^{10} L_{x}^{10 / 3}}\right)\left(\|v\|_{L^{10}[I]}\right. \\
& \left.+\|W\|_{L^{10}[I]}+\|\Gamma\|_{L^{10}[I]}\right)^{3}
\end{aligned}
$$

which by $(5.31),(5.49)$ is bounded by

$$
\begin{aligned}
& \left\|D_{x}^{3 / 5}\left(e^{i\left(t-b_{1}\right) \Delta} \Gamma\left(b_{1}\right)\right)\right\|_{L_{t \in I}^{10} L_{x}^{10 / 3}} \\
& +C \kappa^{1 / 120}\left(\kappa^{1 / 15}+\left\|D_{x}^{3 / 5} W\right\|_{L_{t \in I}^{10} L_{x}^{10 / 3}}\right)^{4} \\
& +C\left(\kappa^{1 / 120}+\left\|D_{x}^{3 / 5} W\right\|_{L_{t \in I}^{10} L_{x}^{10 / 3}}+\left\|D_{x}^{3 / 5} \Gamma\right\|_{L_{t \in I}^{10} L_{x}^{10 / 3}}\right)^{4}\left\|D_{x}^{3 / 5} \Gamma\right\|_{L_{t \in I}^{10} L_{x}^{10 / 3}} .
\end{aligned}
$$


Assume

$$
\left\|D_{x}^{3 / 5}\left(e^{i\left(t-b_{1}\right) \Delta} \Gamma\left(b_{1}\right)\right)\right\|_{L_{t \in I}^{10} L_{x}^{10 / 3}}<\varepsilon<\eta
$$

and

$$
\left\|D_{x}^{3 / 5} W\right\|_{L_{t \in I}^{10} L_{x}^{10 / 3}}<\eta .
$$

Then the bound (5.56), (5.58) clearly implies

$$
\left\|D_{x}^{3 / 5} \Gamma\right\|_{L_{t \in I}^{10} L_{x}^{10 / 3}}<2 \varepsilon+\kappa^{1 / 120} .
$$

From (5.42), we may partition $\mathcal{K}$ in $C(\eta) M_{1}^{10}$ consecutive intervals $I_{1}, I_{2}, \ldots$ such that (5.60) holds for each $I=I_{s}$. When $I=I_{1}, \Gamma\left(b_{1}\right)=\Gamma(b)=0$ and (5.61) gives

$$
\left\|D_{x}^{3 / 5} \Gamma\right\|_{L_{t \in I_{1}}^{10} L_{x}^{10 / 3}}<\kappa_{1} \equiv \kappa^{1 / 120}
$$

In order to verify $(5.59)$ for $I=I_{2}=\left[b_{2}, b_{3}\right]$, write from (5.50)

$$
e^{i\left(t-b_{2}\right) \Delta} \Gamma\left(b_{2}\right)=i \int_{b_{1}}^{b_{2}} e^{i(t-\tau) \Delta}\left[(v+W+\Gamma)|v+W+\Gamma|^{4}-v|v|^{4}-W|W|^{4}\right](\tau) d \tau
$$

and $\left\|D_{x}^{3 / 5}\left(e^{i\left(t-b_{2}\right) \Delta} \Gamma\left(b_{2}\right)\right)\right\|_{L_{t}^{10} L_{x}^{10 / 3}}$ may again be estimated by $(5.52)+\cdots+(5.55)$, $(5.57)+(5.58)$ with $I=\left[b_{1}, b_{2}\right]=I_{1}$, hence by $\varepsilon_{2}=\kappa_{1}$. Thus from (5.61) it follows that

$$
\left\|D_{x}^{3 / 5} \Gamma\right\|_{L_{t \in I_{2}}^{10} L_{x}^{10 / 3}}<2 \varepsilon_{2}+\kappa^{1 / 120}<3 \kappa_{1} \equiv \kappa_{2} .
$$

Also, rewriting (5.56) with $b_{1}$ replaced by $b_{2}$, we get

$$
\begin{aligned}
\left\|D_{x}^{3 / 5}\left(e^{i\left(t-b_{3}\right) \Delta} \Gamma\left(b_{3}\right)\right)\right\|_{L_{t}^{10} L_{x}^{10 / 3} \leq} & \left\|D_{x}^{3 / 5}\left(e^{i\left(t-b_{2}\right) \Delta} \Gamma\left(b_{2}\right)\right)\right\|_{L_{t}^{10} L_{x}^{10 / 3}} \\
& +\kappa^{1 / 120}+\left\|D_{x}^{3 / 5} \Gamma\right\|_{L_{t \in I_{2}}^{6} L_{x}^{10 / 3}} \\
< & \varepsilon_{2}+\kappa_{1}+\kappa_{2}<2 \kappa_{2} \equiv \varepsilon_{3} .
\end{aligned}
$$

The continuation of the process is clear and we get

$$
\left\|D_{x}^{3 / 5} \Gamma\right\|_{L_{t \in I_{s+1}}^{10} L_{x}^{10 / 3}}<2 \varepsilon_{s+1}+\kappa_{1} \text {, hence } \kappa_{s+1}<2 \varepsilon_{s+1}+\kappa_{1}
$$

where

$$
\varepsilon_{s+1}<\varepsilon_{s}+\kappa_{1}+\kappa_{s} .
$$

Hence $\varepsilon_{s}<\kappa_{s}<7^{s} \kappa_{1}$ (provided $<\eta$ ) and consequently

$$
\begin{aligned}
\|\Gamma\|_{L_{x, t}^{10}[\mathcal{K}]} & \leq\left\|D_{x}^{3 / 5} \Gamma\right\|_{L_{t \in \mathcal{K}}^{10} L_{x}^{10 / 3}} \leq \sum_{s<C(\eta) M_{1}^{10}}\left\|D_{x}^{3 / 5} \Gamma\right\|_{L_{t \in I_{s}}^{10} L_{x}^{10 / 3}} \\
& <C(\eta)^{M_{1}^{10}} \kappa^{1 / 120}<1
\end{aligned}
$$

for $\kappa$ sufficiently small.

From (5.42), (5.43), (5.44), (5.68)

$$
\frac{M}{3}-\kappa^{1 / 15} \leq\|w\|_{L_{x, t}^{10}[\mathcal{K}]} \leq\|W\|_{L^{10}[\mathcal{K}]}+\|\Gamma\|_{L^{10}[\mathcal{K}]}<M_{1}+1,
$$

a contradiction. This concludes the proof. 


\section{Conclusion}

The claim verified by induction on the size of the Hamiltonian

$$
H(\phi)=\frac{1}{2} \int|\nabla \phi|^{2}+\frac{1}{6} \int|\phi|^{6}
$$

is that the IVP

$$
\left\{\begin{array}{l}
i u_{t}+\Delta u-u|u|^{4}=0 \\
u(0)=\phi, \phi \text { radial and smooth }
\end{array}\right.
$$

is globally wellposed and satisfies

$$
\|u\|_{L_{x, t}^{10}[I]}<C\left(\|\phi\|_{H^{1}}\right)
$$

for any unit time interval $I$.

The number $\eta>0$ involved in the previous discussion, in particular in (5.7), tends to 0 for $H(\phi) \rightarrow \infty$, a point that should be mentioned. Also, observe that the assumption of radial symmetry only enters that part of the argument related to the use of Morawetz' inequality (which is easier to use in this special case).

Since, by the usual scale invariance of the problem, thus

$$
u_{\lambda}(x, t)=\lambda^{-1 / 2} u\left(\lambda^{-1} x, \lambda^{-2} t\right)
$$

the interval $I$ in (6.3) may be chosen arbitrarily, it follows that

$$
\|u\|_{L_{x, t}^{10}(\mathbb{R})}<C\left(\|\phi\|_{H^{1}}\right) .
$$

Hence also

$$
\left\|D_{x} u\right\|_{L_{x, t}^{10 / 3}(\mathbb{R})}<C\left(\|\phi\|_{H^{1}}\right) .
$$

The role of the smoothness assumption was only to justify certain calculations, in particular those related to Morawetz' inequality. However, since in the conclusion only $H(\phi)$ is involved, it suffices to assume $\phi \in H^{1}$ (and radial). Moreover, properties (6.5) and (6.6) imply scattering (in $H^{1}$ ) (cf. [L-S], [G-V1]) since

$$
\begin{gathered}
\left\|u(t)-e^{i t \Delta}\left(\phi+i \int_{0}^{\infty} e^{-i \tau \Delta}\left(u|u|^{4}\right)(\tau) d \tau\right)\right\|_{H^{1}} \\
=\left\|\int_{t}^{\infty} e^{-i \tau \Delta}\left(u|u|^{4}\right)(\tau) d \tau\right\|_{H^{1}} \stackrel{t \rightarrow \infty}{\rightarrow} 0 .
\end{gathered}
$$

Observe also that if $\phi \in H^{s}, s \geq 1$ (and radial), then

$$
u(t) \in H^{s} \text { for all } t
$$

and

$$
\sup _{t}\|u(t)\|_{H^{s}}<\infty .
$$

One simply performs the IVP-analysis involving the $H^{s}$-norm. Thus, from the integral equation, for $t \in[a, b]=I$

$$
\begin{aligned}
\left\|D_{x}^{s} u\right\|_{L_{x, t}^{10 / 3}[I]} & \leq\left\|D_{x}^{s}\left(e^{i(t-a) \Delta} u(a)\right)\right\|_{L_{x, t}^{10 / 3}}+C\left\|D_{x}^{s} u\right\|_{L_{x, t}^{10 / 3}[I]}\|u\|_{L_{x, t}^{10}[I]}^{4} \\
& \leq C\left\{\|u(a)\|_{H^{s}}+\|u\|_{L^{10}[I]}^{4}\left\|D_{x}^{s} u\right\|_{L^{10 / 3}[I]}\right\} .
\end{aligned}
$$

If we take $I$ such that

$$
\|u\|_{L_{x, t}^{10}[I]} \text { is sufficiently small, }
$$


it follows from (6.9) that

$$
\left\|D_{x}^{s} u\right\|_{L^{10 / 3}[I]} \leq C\|u(a)\|_{H^{s}} .
$$

Also for $t \in I$, from (6.11)

$$
\begin{aligned}
\|u(t)\|_{H^{s}} & \leq\|u(a)\|_{H^{s}}+C \sup _{\|\psi\|_{L^{2}\left(\mathbb{R}^{2}\right)} \leq 1}\left[\left\|e^{i t \Delta} \psi\right\|_{L_{x, t}^{10 / 3}[I]}\left\|D_{x}^{s} u\right\|_{L^{10 / 3}[I]}\|u\|_{L^{10}[I]}^{4}\right] \\
(6.12) & \leq 2\|u(a)\|_{H^{s}} .
\end{aligned}
$$

Observe that by (6.5), $\mathbb{R}_{+}$may be partitioned in intervals $I$ satisfying (6.10), and (6.8) results from iterating (6.9)-(6.12) a bounded number of times.

Theorem. Consider the IVP for the $3 D N L S$

$$
\left\{\begin{array}{l}
i u_{t}+\Delta u-u|u|^{4}=0, \\
u(0)=\phi \in H^{1} \cap H^{s}, s \geq 1,
\end{array}\right.
$$

assuming $\phi$ radial. Then (6.13) is globally wellposed,

$$
\sup _{t}\|u(t)\|_{H^{s}}<C\left(\|\phi\|_{H^{1}},\|\phi\|_{H^{s}}\right)
$$

and there is scattering in $H^{s}$,

$$
\left\|u(t)-e^{i t \Delta}\left(\phi+i \int_{0}^{\infty} e^{-i \tau \Delta}\left(u|u|^{4}\right)(\tau) d \tau\right)\right\|_{H^{s}} \stackrel{t \rightarrow \infty}{\rightarrow} 0 .
$$

\section{Further COMMENTS}

In $4 \mathrm{D}$, the corresponding problem is the IVP

$$
\left\{\begin{array}{l}
i u_{t}+\Delta u-u|u|^{2}=0, \\
u(0)=\phi \in H^{1}\left(\mathbb{R}^{4}\right) \text { and radial }
\end{array}\right.
$$

(the $H^{1}$-critical case is $4 \mathrm{D}$ ).

If one tries to repeat the 3D argument, one encounters a difficulty due to the lower degree nonlinearity (cubic instead of quintic) and some modification is needed.

Observe that it suffices to single out a time interval $I=\left[t_{1}, t_{2}\right], A$ and $N$ such that

$$
\begin{aligned}
\left\|\nabla u\left(t_{1}\right)\right\|_{L^{2}\left[|x|<A N^{-1}\right]} & >\eta_{1}, \\
\|u\|_{L^{6}\left[t_{1} \leq t \leq t_{2}\right]} & <\eta_{2}, \\
\left|t_{2}-t_{1}\right| & >B A^{2} N^{-2},
\end{aligned}
$$

where $\eta_{1}$ is a fixed constant, $\eta_{2}=\eta_{2}\left(\eta_{1}\right)$ is sufficiently small and $B$ is arbitrarily large (in (5.2), the role of the $L^{10}$-norm becomes $L^{6}$-norm as will be clear below).

(A). We first make explicit the estimates involved in the local Cauchy problem. Choose

$$
3 \leq p<4, \quad 3 \leq q<\infty
$$

and define

$$
\frac{1}{2}=\frac{1}{p}+\frac{1}{\bar{p}}=\frac{2}{\bar{p}}+\frac{1}{\bar{q}}, \quad s=\frac{4}{p}+\frac{2}{q}-1 .
$$


Let $t \in[a, b]=I$. The integral equation

$$
u(t)=e^{i(t-a) \Delta} u(a)+i \int_{a}^{t} e^{i(t-\tau) \Delta}\left(u|u|^{2}\right)(\tau) d \tau
$$

implies then, by Strichartz' inequality, the decay-inequality and Young's inequality,

$$
\begin{aligned}
\left\|D_{x}^{s} u(t)\right\|_{L_{t \in I}^{q} L_{x}^{p} \leq} & \left\|D_{x}\left(e^{i(t-a) \Delta} u(a)\right)\right\|_{L_{x, t}^{3}} \\
& +\left\|\int_{a}^{t} \frac{1}{|t-\tau|^{2\left(1-\frac{2}{p}\right)}}\right\| D_{x}^{s}\left(u|u|^{2}\right)\left\|_{L_{x}^{p^{\prime}}} d \tau\right\|_{L_{I}^{q}} \\
& \lesssim\|u(a)\|_{H^{1}}+\left\|\int_{I} \frac{1}{|t-\tau|^{2\left(1-\frac{2}{p}\right)}}\right\| D_{x}^{s} u(\tau)\left\|_{L_{x}^{p}}\right\| u(\tau)\left\|_{L_{x}^{\bar{p}}}^{2} d \tau\right\|_{L^{q}} \\
& \lesssim\|u(a)\|_{H^{1}}+\left\|D_{x}^{s} u\right\|_{L_{I}^{q} L_{x}^{p}\|u\|_{L_{I}^{\bar{q}} L_{x}^{\bar{p}} .}^{2}}
\end{aligned}
$$

For the particular choice $p=3=q$, one gets thus $\bar{p}=6=\bar{q}, s=1$.

(B). Fix $\eta_{0}$ sufficiently small and partition first in intervals $I_{0}$ such that

$$
\|u\|_{L_{x, t}^{6}\left[I_{0}\right]}=\eta_{0}
$$

Hence, from (7.8)

$$
\left\|D_{x} u\right\|_{L^{3}\left[I_{0}\right]}<C .
$$

Choose next a fixed $p<4$ close to 4 . Let $\bar{p}, q=\bar{q}, s$ satisfy (7.6). These parameters are assumed fixed in the sequel. Since from (7.10)

$$
\|u\|_{L_{t \in I_{0}}^{3} L_{x}^{12}}<C
$$

interpolation

$$
\begin{aligned}
\|u\|_{L_{x, t}^{6}\left[I_{0}\right]} & <\|u\|_{L_{t \in I_{0}}^{q} L_{x}^{\bar{p}}}^{\theta}\|u\|_{L_{t \in I_{0}}^{3} L_{x}^{12}}^{1-\theta} \quad\left(\frac{1}{6}=\frac{\theta}{\bar{p}}+\frac{1-\theta}{12}=\frac{\theta}{q}+\frac{1-\theta}{3}\right) \\
& \lesssim\|u\|_{L_{t \in I_{0}}^{q} L_{x}^{\bar{p}}}^{\theta}
\end{aligned}
$$

implies then by (7.9)

$$
\|u\|_{L_{t \in I_{0}}^{q} L_{x}^{\bar{T}}}>\eta_{0}^{1 / \theta}
$$

Choosing $\eta$ a constant $<\eta_{0}^{1 / \theta}$, one further subdivides $I_{0}$ in intervals $I$ such that

$$
\|u\|_{L_{t \in I}^{q} L_{x}^{\bar{p}}} \sim \eta
$$

and hence also, by (7.9), (7.10),

$$
\|u\|_{L^{6}[I]} \leq \eta_{0},\left\|D_{x} u\right\|_{L^{3}[I]}<C .
$$

Interpolating, (7.14), (7.15) imply

$$
\eta \sim\|u\|_{L_{t \in I}^{q} L_{x}^{\bar{p}}} \lesssim\|u\|_{L_{I}^{\infty} L_{x}^{4}}^{1-6 / q}\|u\|_{L^{6}[I]}^{6 / q}<\|u\|_{L_{I}^{\infty} L_{x}^{4}}^{1-6 / q}
$$

and hence there is $t_{0} \in I$ such that

$$
\left\|u\left(t_{0}\right)\right\|_{4}>\eta^{\frac{1}{1-6 / q}}
$$


Write

$$
\left\|u\left(t_{0}\right)\right\|_{4}^{4} \sim \int \sum_{N_{1} \leq N_{2}}\left|\Delta_{N_{1}} u\left(t_{0}\right)\right|^{2}\left|\Delta_{N_{2}} u\left(t_{0}\right)\right|^{2} \lesssim\left(\sup _{N} \frac{\left\|\Delta_{N} u\left(t_{0}\right)\right\|_{\infty}^{2}}{N^{2}}\right)\left\|u\left(t_{0}\right)\right\|_{H^{1}}^{2} .
$$

Thus for some $N_{0}$

$$
\left\|\Delta_{N_{0}} u\left(t_{0}\right)\right\|_{H^{1}} \geq\left\|\Delta_{N_{0}} u\left(t_{0}\right)\right\|_{4} \gtrsim N_{0}^{-1}\left\|\Delta_{N_{0}} u\left(t_{0}\right)\right\|_{\infty} \gtrsim \eta^{\frac{2}{1-6 / q}} .
$$

In fact, the preceding argument permits us clearly to take

$$
N_{0}>\eta^{q / 2}|I|^{-1 / 2} \text {. }
$$

Property (7.19) is preserved for $t$ close enough to $t_{0}$. Define

$$
J=\int\left|\Delta_{N_{0}} u(t)\right|^{4} d x .
$$

Then from the equation (7.1)

$$
\begin{gathered}
\left|\frac{d J}{d t}\right|<\int\left|\Delta_{N_{0}} u(t)\right|^{2}\left|\nabla\left(\Delta_{N_{0}} u(t)\right)\right|^{2}+\int\left|\Delta_{N_{0}} u(t)\right|^{3}\left|\Delta_{N_{0}}\left(u|u|^{2}\right)(t)\right| \lesssim N_{0}^{2}, \\
\left|J(t)-J\left(t_{0}\right)\right| \lesssim\left|t-t_{0}\right| N_{0}^{2}, \\
\left\|\Delta_{N_{0}} u(t)\right\|_{4}^{4}>\left\|\Delta_{N_{0}} u\left(t_{0}\right)\right\|_{4}^{4}-C\left(t-t_{0}\right) N_{0}^{2},
\end{gathered}
$$

and thus

$$
\left\|\Delta_{N_{0}} u(t)\right\|_{4} \gtrsim \eta^{\frac{2}{1-6 / q}} \text { for }\left|t-t_{0}\right| \lesssim \eta^{\frac{8}{1-6 / q}} N_{0}^{-2} .
$$

Since $u$ is radial, (7.21) gives for some $C(\eta)$

$$
\left\|\Delta_{N_{0}} u(t)\right\|_{L^{4}\left[|x|<C(\eta) N_{0}^{-1}\right]} \gtrsim \eta^{\frac{2}{1-6 / q}}
$$

and also

$$
\|u(t)\|_{L^{4}\left[|x|<C(\eta) N_{0}^{-1}\right]} \gtrsim \eta^{\frac{2}{1-6 / q}}>\eta^{3} .
$$

(C). To the interval $I$, associate $t_{0} \in I$ and $N_{0}$ obtained in (B) for which (7.19) and (7.23) hold. Repeating the considerations of section 4 ((7.19) replaces (4.21) and (7.23) replaces (4.11)) and using the 4D-analogue of inequality (2.2)

$$
\int_{I} \int_{|x|<|I|^{1 / 2}} \frac{|u|^{4}}{|x|} d x d t \lesssim C|I|^{1 / 2}
$$

(deduced from Morawetz' inequality), one gets again an interval $I=[a, b], t_{0} \in I$ and $N>c(\eta)|I|^{-1 / 2}$ such that the following properties hold:

$$
\begin{aligned}
\left\|\Delta_{N} u(t)\right\|_{L^{4}\left[|x|<C(\eta) N^{-1}\right]} & \gtrsim \eta^{\frac{2}{1-6 / q}} \text { for }\left|t-t_{0}\right| \lesssim \eta^{\frac{8}{1-6 / q}} N^{-2}, \\
N^{-2} & <\kappa|I|,
\end{aligned}
$$

and $I$ satisfies (7.14), (7.15).

The number $\kappa$ here may be chosen arbitrarily small, provided $\mathcal{J} \supset I$ in (4.1) satisfies

$$
\|u\|_{L_{x, t}^{6}[\mathcal{J}]}>M
$$

where $M$ is taken large enough. 
(D). Assume

$$
|I| \sim b-t_{0}
$$

(the case $|I| \sim t_{0}-a$ is similar).

Fix $\eta_{2}$ in (7.3) and $B$ in (7.4).

One may then clearly get $t_{0}<t_{1}<t_{2}<b$,

$$
t_{1}-t_{0}>\frac{1}{N^{2}}, t_{2}=t_{1}+B^{2} N^{6}\left(t_{1}-t_{0}\right)^{4}
$$

and

$$
\|u\|_{L^{6}\left[t_{1}<t<t_{2}\right]}<\eta_{2}
$$

provided in (7.26)

$$
\kappa<\kappa\left(\eta_{2}, B\right)
$$

Our next purpose is to establish (7.2). Writing

$$
\frac{1}{p}=\frac{1-\varphi}{3}+\frac{\varphi}{\bar{p}}
$$

it follows from (7.6) that $\varphi \lesssim 1$ for $p<4$ close to 4 .

By interpolation, estimate

$$
\left\|D_{x}^{s} u\right\|_{L_{t \in I}^{q} L_{x}^{p}} \leq\|u\|_{L_{I}^{q} L_{x}^{\bar{p}}}^{\varphi}\left\|D_{x}^{s^{\prime}} u\right\|_{L_{I}^{q} L_{x}^{3}}^{1-\varphi}
$$

where

$$
s=(1-\varphi) s^{\prime} .
$$

By (7.15)

$$
\begin{aligned}
\left\|D_{x}^{s^{\prime}} u\right\|_{L_{t \in I}^{q} L_{x}^{3}} & \leq\left\|D_{x} u\right\|_{L_{I}^{3} L_{x}^{3}}^{3 / q}\left\|D_{x}^{1 / 3} u\right\|_{L_{I}^{\infty} L_{x}^{3}}^{1-3 / q} \\
& \leq\left\|D_{x} u\right\|_{L_{x, t}^{3},[I]}^{3 / q}\left\|D_{x} u\right\|_{L_{I}^{\infty} L_{x}^{2}}^{1-3 / q}<C .
\end{aligned}
$$

Substituting (7.35) in (7.33), (7.14) implies

$$
\left\|D_{x}^{s} u\right\|_{L_{I}^{q} L_{x}^{p}} \lesssim \eta^{\varphi} .
$$

Consider $u$ on the interval $\left[t_{0}, t_{1}\right]$. From the integral equation

$$
u(t)=e^{i\left(t-t_{1}\right) \Delta} u\left(t_{1}\right)+i \int_{t}^{t_{1}} e^{i(t-\tau) \Delta}\left(u|u|^{2}\right)(\tau) d \tau .
$$

From the estimate (7.8) and (7.36), (7.14)

$$
\left\|D_{x}^{s}\left[\int_{t}^{t_{1}} e^{i(t-\tau) \Delta}\left(u|u|^{2}\right)(\tau) d \tau\right]\right\|_{L_{I}^{q} L_{x}^{p}} \lesssim\left\|D_{x}^{s} u\right\|_{L_{I}^{q} L_{x}^{p}}\|u\|_{L_{I}^{q} L_{x}^{\bar{p}}}^{2}<\eta^{2+\varphi} .
$$

From (7.25), it follows that for $\left|t-t_{0}\right|<\eta^{\frac{8}{1-6 / q}} N^{-2}$

$$
\left\|\Delta_{N} u(t)\right\|_{L^{p}\left[|x|<C(\eta) N^{-1}\right]}>N^{1-\frac{4}{p}}\left\|\Delta_{N} u(t)\right\|_{L^{4}\left[|x|<C(\eta) N^{-1}\right]}^{4 / p}>N^{1-4 / p} \eta^{\frac{8}{p(1-6 / q)}} .
$$

Defining

$$
\Omega=\left[|x|<C(\eta) N^{-1} ; t_{0}<t<t_{0}+\eta^{\frac{8}{1-6 / q}} N^{-2}\right]
$$


(7.39), (7.6) then imply that for $p$ close to 4

$$
N^{s}\left\|\left.\Delta_{N} u\right|_{\Omega}\right\|_{L_{t}^{q} L_{x}^{p}}>N^{s-\frac{2}{q}+1-4 / p} \eta^{\frac{8}{q-6}+\frac{8}{p(1-6 / q)}}=\eta^{2+} .
$$

Comparing (7.38), (7.40), one deduces from (7.37) that

$$
N^{s}\left\|\left.e^{i\left(t-t_{1}\right) \Delta} \Delta_{N} u\left(t_{1}\right)\right|_{\Omega}\right\|_{L_{t}^{q} L_{x}^{p}}>\eta^{2+}>\eta^{3} .
$$

In order to relate the information (7.41) to $u\left(t_{1}\right)$, the following lemma is used.

Lemma 7.42. Assume $\|\phi\|_{H^{1}}<C$ and

$$
N^{s}\left\|e^{i t \Delta} \Delta_{N} \phi\right\|_{L_{\left[0<t<Q / N^{2}\right]}^{q} L_{[|x|<Q / N]}^{p}>\delta}(Q>10) .
$$

Then

$$
\|\nabla \phi\|_{L^{2}\left[|x|<\frac{C(\delta) Q^{2}}{N}\right]}>\frac{\delta}{2} .
$$

Proof. Let $\psi=\Delta_{N} \phi$. Then

$$
\left(e^{i t \Delta} \psi\right)(x)=\int_{|\xi| \sim N} \widehat{\psi}(\xi) e^{i\left(x \xi+t \xi^{2}\right)} d \xi=\left(\psi * K_{t}\right)(x)
$$

where we define

$$
K_{t}(x)=\int \gamma\left(\frac{\xi}{N}\right) e^{i\left(x \xi+t \xi^{2}\right)} d \xi
$$

and $0 \leq \gamma \leq 1$ a standard bump function, $\gamma(y)=1$ for $|y|<2$ and $\gamma(y)=0$ for $|y|>3$. Thus from (7.46), one may ensure an estimate

$$
\left|K_{t}(x)\right| \lesssim\left(\frac{N^{-1}+|t| N}{|x|}\right)^{10} N^{4}
$$

and for $|t|<\frac{Q}{N^{2}}$

$$
\left|K_{t}(x)\right| \lesssim\left(\frac{Q}{N|x|}\right)^{10} N^{4}
$$

Take

$$
R=\delta^{-1} Q^{2}
$$

and observe from (7.48) that

$$
\left\|K_{t}\right\|_{L^{1}[|x|>R / N]} \lesssim \frac{Q^{10}}{R^{6}}
$$

Write by (7.45)

$$
\begin{aligned}
& \delta<N^{s}\left\|e^{i t \Delta} \psi\right\|_{L_{0<t<\frac{Q}{N^{2}}}^{q} L_{|x|<\frac{Q}{N}}^{p} \leq} \leq \\
& N^{s}\left\|\psi *\left(\left.K_{t}\right|_{|y|<R N^{-1}}\right)\right\|_{L_{|t|<\frac{Q}{N^{2}}} L_{|x|<\frac{Q}{N}}^{p}} \\
& +N^{s}\left\|\psi *\left(\left.K_{t}\right|_{|y|>R N^{-1}}\right)\right\|_{L_{|t|<\frac{Q}{N^{2}}}^{q} L_{x}^{p}}
\end{aligned}
$$

and since $R>Q$

$$
(7.51) \leq N^{s}\left\|\left(\psi \gamma_{1}\right) *\left(\left.K_{t}\right|_{|y|<R N^{-1}}\right)\right\|_{L_{|t|<\frac{Q}{N^{2}}}} L_{x}^{p}
$$


with

$$
\gamma_{1}(x)=\gamma\left(\frac{N}{R} x\right)
$$

Estimate

$$
\begin{aligned}
(7.53) \leq & N^{s}\left\|\left(\psi \gamma_{1}\right) * K_{t}\right\|_{L_{t}^{q} L_{x}^{p}} \\
& +N^{s}\left\|\left(\psi \gamma_{1}\right) *\left(\left.K_{t}\right|_{|y|>R N^{-1}}\right)\right\|_{L_{|t|<\frac{Q}{N^{2}}} L_{x}^{p} .}
\end{aligned}
$$

Next, by (7.50), (7.6)

$$
\begin{aligned}
(7.52),(7.56) & \lesssim N^{s} \frac{Q^{10}}{R^{6}}\left(\frac{Q}{N^{2}}\right)^{1 / q}\|\psi\|_{L_{x}^{p}} \\
& \lesssim N^{s} \frac{Q^{10}}{R^{6}}\left(\frac{Q}{N^{2}}\right)^{1 / q} N^{-1+4\left(\frac{1}{2}-\frac{1}{p}\right)} \lesssim \frac{Q^{11}}{R^{6}}<\frac{\delta}{Q}
\end{aligned}
$$

Define

$$
\frac{1}{r}=\frac{\frac{1}{p}-\frac{1}{q}}{1-\frac{3}{q}}
$$

Interpolating,

$$
\|\|_{L_{t}^{q} L_{x}^{p}} \leq\|\|_{L_{x, t}^{3}}^{3 / q}\|\|_{L_{t}^{\infty} L_{x}^{r}}^{1-3 / q}
$$

Strichartz' inequality gives the bound

$$
\begin{aligned}
(7.55) & \lesssim N^{s}\left\|\psi \gamma_{1}\right\|_{2}^{3 / q}\left\|\psi \gamma_{1}\right\|_{r}^{1-3 / q} \\
& \lesssim N^{3+4\left(1-\frac{3}{q}\right)\left(\frac{1}{2}-\frac{1}{r}\right)}\left\|\psi \gamma_{1}\right\|_{2} \leq N\left\|\Delta_{N} \phi\right\|_{\left.L^{2}|x|<\frac{3 R}{N}\right]}
\end{aligned}
$$

by $(7.58),(7.6),(7.54)$.

Collecting estimates, we conclude that

$$
N\left\|\Delta_{N} \phi\right\|_{L^{2}\left[|x|<3 \delta^{-1} Q^{2} N^{-1}\right]}>\frac{\delta}{2}
$$

from which (7.44) is easily deduced.

Coming back to (7.41), apply the lemma with $\phi=u\left(t_{1}\right), \delta=\eta^{3}, Q=C(\eta)+$ $\left(t_{1}-t_{0}\right) N^{2}$. (7.44) gives then that

$$
\left\|\nabla u\left(t_{1}\right)\right\|_{L^{2}\left[|x|<C(\eta)\left(t_{1}-t_{0}\right)^{2} N^{3}\right]}>\frac{\eta^{3}}{2},
$$

which is (7.2) with $\eta_{1}=\frac{\eta^{3}}{2}, A=C(\eta)\left(t_{1}-t_{0}\right)^{2} N^{4}$. Conditions (7.3), (7.4) are clearly satisfied by (7.29), (7.30).

\section{REFERENCES}

[L-S] J. Lin, W. Strauss, Decay and scattering of solutions of a nonlinear Schrödinger equation, JFA Vol. 30, no. 2 (1978), 245-263. MR 80k:35056

[Str] M. Struwe, Globally regular solutions to the $u^{5}-$ Klein-Gordon equations, Ann. Scuola Norm Sup. Pisa, Ser. 4, 15 (1988), 495-513. MR 90j:35142

[Gr] M. Grillakis, Regularity and asymptotic behavior of the wave equation with a critical nonlinearity, Annals Math. 132 (1990), 485-509. MR 92c:35080

[S-S] J. Shatah, M. Struwe, Regularity results for nonlinear wave equations, Annals of Math. 138 (1993), 503-518. MR 95f:35164

[Caz] T. Cazenave, An introduction to nonlinear Schrödinger equations, Textos de Metodes Matematicos 26 (Rio de Janeiro). 
[C-W] T. Cazenave, F. Weissler, The Cauchy problem for the critical nonlinear Schrödinger equation in $H^{s}$, Nonlinear Anal., TMA 14 (1990), 807-836. MR 91j:35252

[G-V1] J. Ginibre, G. Velo, Scattering theory in the energy space for a class of nonlinear Schrödinger equations, J. Math Pure Appl. 64 (1985), 363-401. MR 87i:35171

[G-V2] J. Ginibre, G. Velo, On a class of nonlinear Schrödinger equations with nonlocal interaction, Math. Z. 170 (1980), 109-136. MR 82c:35018

[S] R. Strichartz, Restrictions of Fourier transforms to quadratic surfaces and decay of solutions of wave equations, Duke Math. J. 44 (1977), 705-714. MR 58:23577

School of Mathematics, Institute for Advanced Study, Princeton, New Jersey 08540

E-mail address: bourgain@math.ias.edu 